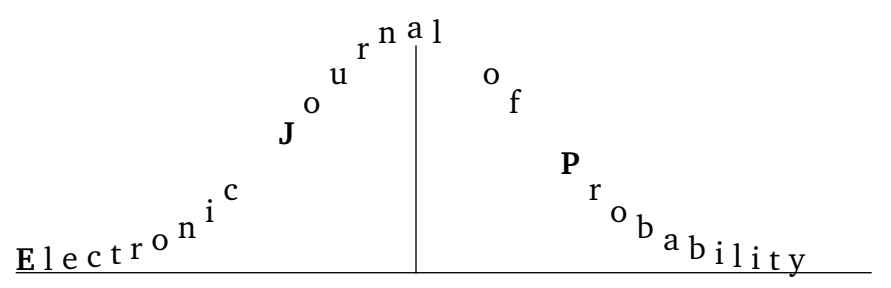

Vol. 15 (2010), Paper no. 37, pages 1161-1189.

Journal URL

http://www.math.washington.edu/ ejpecp/

\title{
The Green Functions \\ Of Two Dimensional Random Walks Killed On A Line And Their Higher Dimensional Analogues
}

\author{
Kôhei UCHIYAMA \\ Department of Mathematics \\ Tokyo Institute of Technology \\ Oh-okayama, Meguro Tokyo, 152-8551, Japan \\ uchiyama@math.titech.ac.jp
}

\begin{abstract}
We obtain asymptotic estimates of the Green functions of random walks on the two-dimensional integer lattice that are killed on the horizontal axis. A basic asymptotic formula whose leading term is virtually the same as the explicit formula for the corresponding Green function of Brownian motion is established under the existence of second moments only. Some refinement of it is given under a slightly stronger moment condition. The extension of the results to random walks on the higher dimensional lattice is also given.
\end{abstract}

Key words: asymptotic formula, Green function, random walk of zero mean and finite variances, absorption on a line.

AMS 2000 Subject Classification: Primary 60G50; Secondary: 60J45.

Submitted to EJP on October 5, 2009, final version accepted May 19, 2010. 


\section{Introduction and Results}

Let $S_{n}^{x}=x+\xi_{1}+\cdots+\xi_{n}$ be a random walk on $\mathbf{Z}^{2}$ (the two dimensional integer lattice) starting at $S_{0}^{x}=x \in \mathbf{Z}^{2}$. Here $\xi_{j}, j=1,2, \ldots$, are independent and identically distributed $\mathbf{Z}^{2}$-valued random variables defined on a probability space $(\Omega, \mathscr{F}, P)$. The walk $S_{n}^{0}$ is supposed irreducible and having zero mean and finite variances. Let $\left[x_{1}, x_{2}\right]$ stand for a point of $\mathbf{R}^{2}$ with components $x_{1}$ and $x_{2}$ and put

$$
L=\{[s, 0]: s \in \mathbf{R}\} \quad \text { (the first coordinate axis). }
$$

In this paper we obtain asymptotic estimates of the Green function $G_{L}$ of the walk killed on $L$ :

$$
G_{L}(x, y)=\sum_{n=0}^{\infty} p_{L}^{n}(x, y)
$$

where $p_{L}^{n}(x, y)=P\left[S_{n}^{x}=y, S_{j}^{x} \notin L\right.$ for $\left.j=1, \ldots, n-1\right]$, the $n$-step transition probability of the killed walk; in particular $p_{L}^{0}(x, y)=\delta_{x, y}$ (Kronecker's symbol). Note that $p_{L}^{n}(x, y)$ (with $n \geq 1$ ), hence $G_{L}(x, y)$, may be positive even if $x, y \in L$. Our definition, thus different from usual one (but only if $|x| \cdot|y|=0$ ), is convenient when duality relations are considered (see Remark 4 below). Let $X$ and $Y$ be the first and second component of $\xi_{1}=S_{1}^{0}$, respectively, put $\sigma_{1}^{2}=E X^{2}, \sigma_{12}=E X Y, \sigma_{2}^{2}=E Y^{2}$, $\sigma_{j j}=\sigma_{j}^{2}(j=1,2), Q=\left(\sigma_{i j}\right)$ (the covariance matrix of $\left.S_{1}^{0}\right)$ and $\sigma=|\operatorname{det} Q|^{1 / 4}$, and define the norm $\|x\|, x \in \mathbf{R}^{2}$ by $\|x\|^{2}=\sigma^{2} Q^{-1}(x)$, where $Q^{-1}$ and $Q^{-1}(x)$ stand for the inverse matrix of $Q$ and its quadratic form, respectively.

For $a, b \in \mathbf{R}, a \vee b$ and $a \wedge b$ denote, respectively, the maximum and the minimum of $a$ and $b$. The function $t \log t$ is understood continuously extended to $t=0$.

Theorem 1.1. Suppose that $n k>0$ and let $B_{s, n, k}$ be given via the equation

$$
G_{L}([0, n],[s, k])=\frac{1}{2 \pi \sigma^{2}} \log \left(1+\frac{4\left(\sigma / \sigma_{2}\right)^{2} n k}{\|[s, k-n]\|^{2} \vee 1}\right)+B_{s, n, k} .
$$

Then

$$
B_{s, n, k}=o\left(1 \vee \log \left(\frac{n k}{\|[s, k-n]\|^{2} \vee 1}\right)\right) \quad \text { as } n k \rightarrow \infty
$$

uniformly in $s$; in particular $B_{s, n, k}=o(\log n)$ as $n \rightarrow \infty$ uniformly in $s, k$. Moreover $B_{s, n, k}$ is uniformly bounded if and only if $E\left[\left|S_{1}^{0}\right|^{2} \log \left|S_{1}^{0}\right|\right]<\infty$ and if this is the case $B_{s, n, k} \rightarrow 0$ as $\|[s, k-n]\| \rightarrow \infty$.

Theorem 1.2. Suppose that $n k<0$. Then $G_{L}([0, n],[s, k]) \rightarrow 0$ as $\|[s, k-n]\| \rightarrow \infty$.

For each pair of $k, n, G_{L}([0, n],[s, k]) \rightarrow 0$ as $|s| \rightarrow \infty$ and $G_{L}$ is everywhere positive. With this taken into account it is inferred from the first half of Theorem 1.1 that under the condition $n k>0$, $G_{L}$ is bounded away from zero if and only if so is $n k /\left(\|[s, k-n]\|^{2} \vee 1\right)$. Unless this is the case the estimate in Theorem 1.1 is crude. The next result is complementary in this respect (its proof is much more involved than that of Theorem 1.1).

Let $a(n)$ ( $n \in \mathbf{Z}^{2}$ ) denote the potential function of the one dimensional random walk of the second component of $S_{n}^{0}: a(n)=\sum_{k=0}^{\infty}\left(P\left[S_{k}^{0} \in \mathbf{Z} \times\{0\}\right]-P\left[S_{k}^{0} \in \mathbf{Z} \times\{-n\}\right]\right)$. (Cf. [7]:T29.1.) Also put $a^{*}(n)=\delta_{0, n}+a(n)$

$$
\mu=\sigma_{12} / \sigma_{2}^{2} \quad \text { and } \quad \tilde{X}=X-\mu Y .
$$


Theorem 1.3. If $E\left[\tilde{X}^{2} \log |\tilde{X}|\right]<\infty$, then, as $\left.(k \vee 1)(n \vee 1)\right) /\left(\|[s, k-n]\|^{2} \vee 1\right) \rightarrow 0$

$$
G_{L}([0, n],[s, k])=\frac{1}{\pi} \frac{\sigma_{2}^{4} a^{*}(n) a^{*}(-k)+n k}{\sigma_{2}^{2}\|[s, k-n]\|^{2}}+o\left(\frac{(n \vee 1)(|k| \vee 1)}{\|[s, k-n]\|^{2}}\right) \text { for } n \geq 0, k, s \in \mathbf{Z}
$$

REMARK 1. Let $\lambda=\sigma^{2} / \sigma_{2}^{2}$ and observe that

$$
\|[s, m]\|^{2}=\lambda^{-1}(s-\mu m)^{2}+\lambda m^{2}=\|[s+2 \mu m,-m]\|^{2}
$$

and $\|[s, k-n]\|^{2}+4 \lambda k n=\|[s+2 \mu n, k+n]\|^{2}$. Then the formula of Theorem 1.1 is understood to be in accordance with the one for Brownian motion, say $g^{\circ}(a, b)$, that is explicit owing to reflection principle (see Appendix (B)). Donsker's invariance principle implies that if two points $a, b$ are taken from the upper half plane $\mathbf{R} \times(0, \infty), \varepsilon>0$ and $\alpha_{x}=|a| /|x|\left(x \in \mathbf{Z}^{2}\right)$, then $\alpha_{x}^{2} \sum_{\left\{y:\left|\alpha_{x} y-b\right|<\varepsilon\right\}} G_{L}(x, y)$ converges to $\int_{|u-b|<\varepsilon} g^{\circ}(a, u) d u$ as $|x| \rightarrow \infty$ in such a way that $\lim \alpha_{x} x=a$, but not much more.

Remark 2. For $n<0$, the formula of Theorem 1.3 hold true as $n[(-k) \vee 1] /\|[s, k-n]\|^{2} \rightarrow 0$ by duality. In the case when $n k<0$ and $|k| \wedge|n| \rightarrow \infty$ we have $\sigma_{2}^{4} a(n) a(-k)+n k=o(n k)$, so that any proper asymptotic form of $G_{L}$ is not given by it; the determination of it requires more detailed analysis than that carried out in this paper and will be made in a separate paper ([11]).

REMARK 3. It follows from (1.1) that under the constraint $s^{2}+(n-k)^{2}>\varepsilon n k>0$ (for some $\varepsilon>0$ ) the convergence to zero of $B_{s, n, k}$ stated in Theorem 1.1 holds true without assuming $E\left[\left|S_{1}^{0}\right|^{2} \log \left|S_{1}^{0}\right|\right]<$ $\infty$, which however cannot be removed in general (see also Theorem 3.2). Similarly the moment condition $E\left[\tilde{X}^{2} \log |\tilde{X}|\right]<\infty$ in Theorem 1.3 is needed only in the case when $|k|+|n|=o(|s|)$ (Lemmas 4.2 and 4.3$)$, while without it $\limsup _{|s| \rightarrow \infty} s^{2} G_{L}([0, n],[s, k])$ can be infinite in view of Theorem 1.3 of [9].

REMARK 4. If $\tilde{G}_{L}$ denotes the Green function associated with the dual process (i.e. the process $\left.\left(-S_{n}^{-x}\right)\right)$, then $\tilde{G}_{L}([0, n],[s, k])=G_{L}([0, k],[-s, n])$. Because of this duality we may suppose that $|k| \leq n$ for the proof of Theorems above. In view of (1.4) (that also follows from the duality) the results on $H_{[0, n]}(s)$ obtained in [9] provide the formula of Theorem 1.3 in the case $n k=0$. Thus we may further suppose that $|k|>0$.

This paper is in a sense a continuation of [9] in which the hitting distribution of $L$ for the walk starting at $[0, n]$ is evaluated. As in [9] put

$$
\psi(t, l)=E\left[e^{i t X+i l Y}\right]
$$

and for $t \neq 0$,

$$
\pi_{k}(t)=\frac{1}{2 \pi} \int_{-\pi}^{\pi} \frac{1}{1-\psi(t, l)} e^{-i k l} d l \quad \text { and } \quad \rho(t)=\frac{1}{\pi_{0}(t)} .
$$

Let $H_{x}(s)$ denote the probability that the first entrance after time 0 of the walk $S_{n}^{x}$ into $L$ takes place at $[s, 0]: H_{x}(s)=P\left[\right.$ for some $\tau \geq 1, S_{\tau}^{x}=[s, 0]$ and $S_{n}^{x} \notin L$ for $\left.0<n<\tau\right]$. The evaluation of $H_{x}(s)$ in [9] is based on the Fourier representation formula

$$
H_{[0, k]}(s)=\frac{1}{2 \pi} \int_{-\pi}^{\pi} \rho(t)\left[\pi_{-k}(t)-\delta_{0, k}\right] e^{-i s t} d t,
$$


while our starting point for evaluation of $G_{L}$ is

$$
G_{L}([0, n],[s, k])=\frac{1}{2 \pi} \int_{-\pi}^{\pi}\left[\pi_{-n+k}(t)-\rho(t)\left(\pi_{-n}(t)-\delta_{0, n}\right) \pi_{k}(t)\right] e^{-i s t} d t,
$$

which is derived in a standard way (see Appendix (A) for a proof). From (1.2) and (1.3) it follows that

$$
G_{L}([0,0], y)=H_{-y}(0)
$$

The asymptotic formulae presented above are extended to higher dimensional walks. Consider a $d$-dimensional random walk $S_{n}^{0}$, moving on $\mathbf{Z}^{d}$, for $d \geq 3$. Suppose that the walk is irreducible and has zero mean and the finite variances. Let $X$ and $Y$ be the first $\mathbf{Z}^{d-1}$ component and the $d$-th component of $S_{1}^{0}$, respectively. Put $\sigma_{d}^{2}=E\left[Y^{2}\right]$ and define the norm $\|\cdot\|$ analogously to the two dimensional case. For $k \in \mathbf{Z}, x=\left(x_{1}, \ldots, x_{d-1}\right) \in \in \mathbf{Z}^{d-1}$, let $[x, k]$ stand for the $d$-dimensional point $\left(x_{1}, \ldots, x_{d-1}, k\right)$. Theorems 1.3 is extended as follows.

Theorem 1.4. Suppose the moment conditions $E\left[|X|^{d}\right]<\infty$ and

$$
E\left[Y^{2} \log |Y|\right]<\infty \text { for } d=3 \text { and } E\left[|Y|^{d-1}\right]<\infty \text { for } d \geq 4
$$

to hold. Then for $n \geq 0, k \in \mathbf{Z}, x \in \mathbf{Z}^{d-1}$, as $([k \vee 0] n+1) /\left(\|[x, k-n]\|^{2} \vee 1\right) \rightarrow 0$

$$
G_{L}([0, n],[x, k])=\frac{\Gamma(d / 2)}{\pi^{d / 2}} \cdot \frac{\sigma_{d}^{4} a^{*}(n) a^{*}(-k)+n k}{\sigma_{d}^{2}\|[x, k-n]\|^{d}}+o\left(\frac{(n \vee 1)(|k| \vee 1)}{\|[x, k-n]\|^{d}}\right) .
$$

Let $p^{m}(x, y)=P\left[S_{m}^{x}=y\right]$ and $G(x, y):=\sum_{m=0}^{\infty} p^{m}(x, y)$, the Green function of the walk $S_{n}^{x}$. In the next theorem we give results corresponding to Theorems 1.1 and 1.2 under the following moment condition (to be a minimal one for the obtained estimates):

$$
E\left[Y^{2} \log |Y|\right]<\infty \text { if } d=4 \text { and } E\left[|Y|^{d-2}\right]<\infty \text { if } d \geq 5 .
$$

Theorem 1.5. Suppose (1.6) to hold. Then as $|n| \vee|k| \vee|x| \rightarrow \infty$ in such a way that $|n k| /\|[x, k-n]\|^{2}$ is bounded away from zero,

$$
\begin{aligned}
& G_{L}([0, n],[x, k]) \\
& \quad= \begin{cases}G([0, n],[x, k])-\kappa_{d} \sigma^{-2}\|[x+2 n \mu, k+n]\|^{-d+2}(1+o(1)) & \text { if } n k>0, \\
o\left(\|[x, k-n]\|^{-d+2}\right) & \text { if } n k<0,\end{cases}
\end{aligned}
$$

where $\mu=E[Y X]\left(\in \mathbf{R}^{d-1}\right)$ and $\kappa_{d}=\Gamma(d / 2) /(d-2) \pi^{d / 2}$.

REMARK 5. The Fourier representation (1.3) is a manifestation of the decomposition

$$
G_{L}([0, n],[x, k])=G([0, n],[x, k])-\sum_{j \in \mathbf{Z}} H_{[0, n]}(j) G([j, 0],[x, k]) .
$$

As for the two-dimensional walk, if $A(x, y)=\sum_{m=0}^{\infty}\left[p^{m}(0,0)-p^{m}(x, y)\right]$, then as a two dimensional analogue of (1.8), with $-A(x, y)$ replacing $G(x, y)$, we have

$$
G_{L}([0, n],[s, k])=-A([0, n],[s, k])+\sum_{j \in \mathbf{Z}} H_{[0, n]}(j) A([j, 0],[s, k]) .
$$


If the walk satisfies a certain condition concerning symmetry and continuity in the vertical direction these identities reduce to the reflection principle. Otherwise, however, direct computation using them together with the estimates of $H_{[0, n]}$ and $G$ (as found e.g. in [9], [8]) does not give any correct asymptotic form of $G_{L}$ unless either the first term of the decomposition is dominant or we have nice estimates of the second order terms of $H_{[0, n]}$ and $G$; in any case the results obtained in such a way are in general not sharp.

Two relevant matters on some closely related random walks are briefly discussed in the rest of this section.

Walks Killed on a half horizontal axis. Put $L^{ \pm}=\{[s, 0]: \pm s \geq 0\} \subset \mathbf{Z}^{2}$ (the non-negative and nonpositive parts of the horizontal axis) and let $G_{L^{-}}(x, y)$ be the Green function of the two dimensional walk killed on $L^{-}$that is defined analogously to $G_{L}(x, y)$. Then it follows that for $y \notin L^{-}$,

$$
G_{L^{-}}(x, y)=G_{L}(x, y)+\sum_{s=1}^{\infty} H_{x}(s) G_{L^{-}}([s, 0], y)
$$

and if $y=[s, 0] \in L^{-}$,

$$
G_{L^{-}}(x,[s, 0])=\sum_{s_{1}=1}^{\infty} H_{x}\left(s_{1}\right) g_{(-\infty, 0]}\left(s_{1}, s\right),
$$

where $g_{(-\infty, 0]}\left(s, s_{1}\right)$ denotes the Green function of one dimensional walk which is the trace on $L^{+} \backslash\{0\}$ of the walk $\left(S_{n}^{[s, 0]}\right)$ killed on $L^{-}$. Consider the last identity for the time-reversed walk. Indicating the dual objects by putting $\sim$ over the notation as in Remark 4, we then find the identities $G_{L^{-}}(x, y)=\tilde{G}_{L^{-}}(y, x), \tilde{H}_{y}\left(s_{1}\right)=H_{-y}\left(-s_{1}\right)$ and $\tilde{g}_{(-\infty, 0]}\left(s_{1}, s\right)=g_{(-\infty, 0]}\left(s, s_{1}\right)$ and substitute these into (1.10) to obtain that

$$
G_{L^{-}}(x, y)=G_{L}(x, y)+\sum_{s=1}^{\infty} \sum_{s_{1}=1}^{\infty} H_{x}(s) H_{-y}\left(-s_{1}\right) g_{(-\infty, 0]}\left(s, s_{1}\right) \quad\left(y \notin L^{-}\right) ;
$$

similarly

$$
G_{L^{-}}([s, 0], y)=\tilde{G}_{L^{-}}(y,[s, 0])= \begin{cases}H_{-y}^{+}(-s), & s \leq 0, \\ \sum_{s_{1}=1}^{\infty} H_{-y}\left(-s_{1}\right) g_{(-\infty, 0]}\left(s, s_{1}\right) & s>0,\end{cases}
$$

where $H_{x}^{+}(s)$ denotes the entrance distribution into $L^{+}$(defined analogously to $H_{x}(s)$ ).

Certain asymptotic estimates of $H_{x}^{+}(s)$ are obtained in [10] (under the present setting), [4] (for simple walk) and [1] (for a class of random walks with a finite range of jump by an algebraic method). Computation made in Section 4 of [10] would be helpful for evaluation of the double sum in (1.11).

Walks Killed on a half plane. Let $D=\left\{[s, k] \in \mathbf{Z}^{2}: k \leq 0\right\}$, the lower half plane. The evaluation of $G_{L}$ is intimately related to that of $G_{D}$, the Green function of the walk killed on $D$. First it is pointed out that if the walk is either upwards or downwards continuous, namely either $P[Y \geq 2]=0$ or $P[Y \leq-2]=0$, then $G_{L}$ and $G_{D}$ agree on $\mathbf{Z}^{2} \backslash D$. Let $f^{+}(n)$ (resp. $\left.f^{-}(n)\right)(n=1,2, \ldots)$ be the positive harmonic function that is asymptotic to $n$ of the one dimensional walk killed on $\{n \leq 0\}$ 
whose increment has the same law as $Y$ (resp. $-Y): f_{ \pm}(n)=E\left[f_{ \pm}(n \pm Y) ; n \pm Y>0\right](n \geq 1)$ and $\lim _{n \rightarrow \infty} f_{ \pm}(n) / n=1$, which each exists uniquely ([7]:p.212). If $P[Y \geq 2]=0$, then for $n \geq 1$,

$$
f_{+}(n)=\frac{1}{2}\left(\sigma_{2}^{2} a(n)+n\right) \quad \text { and } \quad f_{-}(n)=\sigma_{2}^{2} a(-n)=n,
$$

and the formula of Theorem 1.3 may be written as

$$
G_{D}([0, n],[s, k])=\frac{2 f_{+}(n) f_{-}(k)}{\pi \sigma_{2}^{2}\|[s, k-n]\|^{2}}(1+o(1))
$$

$\left(n, k>0, n k /\|[s, k-n]\|^{2} \rightarrow 0\right)$. In a separate paper [11] we prove 11.12 to be true in general under $E\left[\tilde{X}^{2} \log |\tilde{X}|\right]<\infty$, where the proof rests on Theorem 1.3 of the present paper.

The rest of the paper is organized as follows. In Section 2 some preliminary lemmas are established. Theorems 1.1 and 1.2 are proved in Section 3 and Theorem 1.3 in Section 4. In Section 5 their $d$ dimensional analogues, i.e., Theorems 1.4 and 1.5, are proved. Section 6 , the last section, consists of Appendices (A), (B), (C) and (D) ; in (A) a proof of the Fourier representation formula (1.3) is given; we exhibit in (B) the formula for the Green function of Brownian motion corresponding to $G_{L}$ for comparison with those given above and provide in (C) certain details for the case $\sigma_{12} \neq 0$ which are omitted in Section 2; in (D) two lemmas concerning Fourier type integrals are proved.

\section{Preliminary Lemmas}

Throuout the rest of the paper we suppose that $\sigma_{12}=0$ unless otherwise stated explicitly, so that the quadratic form $Q(\theta)=Q\left(\theta_{1}, \theta_{2}\right)=\sum \sigma_{i j} \theta_{i} \theta_{j}\left(\theta=\left(\theta_{1}, \theta_{2}\right) \in \mathbf{R}^{2}\right)$ is of the form

$$
Q(\theta)=\sigma_{1}^{2} \theta_{1}^{2}+\sigma_{2}^{2} \theta_{2}^{2}
$$

The case $\sigma_{12} \neq 0$ can be similarly treated and necessary modifications will be given in Appendix (C).

Let $n>0$. As an ideal substitute for $\pi_{-n}(t)$, we bring in

$$
\pi_{-n}^{\circ}(t)=\frac{1}{2 \pi} \int_{\mathbf{R}} \frac{2}{Q(t, l)} e^{i n l} d l
$$

It follows that

$$
\pi_{-n}^{\circ}(t)=\frac{e^{-n \lambda|t|}}{\sigma^{2}|t|}
$$

where, as in Remark 1,

$$
\lambda=\sigma_{1} / \sigma_{2}=\sigma^{2} / \sigma_{2}^{2}
$$

On writing $m=n-k$ the Fourier representation $(1.3)$ is decomposed as

$$
G_{L}([0, n],[s, k])=\frac{1}{2 \pi} \int_{-\pi}^{\pi}\left(\pi_{-m}(t)-\pi_{-n}(t)\right) e^{-i s t} d t+\frac{1}{2 \pi} \int_{-\pi}^{\pi} \frac{\left(\pi_{0}(t)-\pi_{k}(t)\right) \pi_{-n}(t)}{\pi_{0}(t)} e^{-i s t} d t .
$$

We evaluate these two integrals separately in Propositions 2.1 and 2.2 given below. 
For the evaluation of the first integral above we observe that

$$
\begin{aligned}
\frac{1}{2 \pi} \int_{\mathbf{R}}\left(\pi_{-m}^{\circ}(t)-\pi_{-n}^{\circ}(t)\right) e^{-i s t} d t & =\frac{1}{\pi \sigma^{2}} \int_{0}^{\infty} \frac{e^{-|m| t / \lambda}-e^{-|n| t / \lambda}}{t} \cos s t d t \\
& =\frac{1}{2 \pi \sigma^{2}} \log \left(\frac{s^{2}+\lambda^{2} n^{2}}{s^{2}+\lambda^{2} m^{2}}\right) .
\end{aligned}
$$

Lemma 2.1. Define $J=J_{s, n, m}$ by

$$
J=\frac{1}{(2 \pi)^{2}} \int_{-\pi}^{\pi} e^{-i s t} d t \int_{-\pi}^{\pi} \frac{2}{Q(t, l)}\left(e^{i m l}-e^{i n l}\right) d l-\frac{1}{2 \pi \sigma^{2}} \log \left(\frac{s^{2}+\lambda^{2} n^{2}}{s^{2}+\lambda^{2} m^{2}}\right) .
$$

Then $|J| \leq \frac{C}{\left(s^{2}+1\right)\left(n^{2} \wedge m^{2}+1\right)}$ if $s^{2}+\left(m^{2} \wedge n^{2}\right) \neq 0$.

Proof. Write $J=-2 \pi^{-2}(I+I I)$, where

$$
I:=\int_{\pi}^{\infty} \cos s t d t \int_{0}^{\pi} \frac{\cos m l-\cos n l}{Q(t, l)} d l \text { and } I I:=\int_{0}^{\infty} \cos s t d t \int_{\pi}^{\infty} \frac{\cos m l-\cos n l}{Q(t, l)} d l .
$$

Then $I \leq C /\left(s^{2}+1\right)\left(m^{2} \wedge n^{2}+1\right)$ since the function $\int_{\pi}^{\infty}[Q(t, l)]^{-1} \cos s t d t$ is a nice smooth function of $l$ bounded by $C^{\prime} /\left(s^{2}+1\right)$; the same or a rather better bound is true for $I I$ as readily seen from the identity $I I=\sigma^{-2} \int_{\pi}^{\infty} \frac{\cos m l-\cos n l}{l} e^{-|s| l / \lambda} d l$.

Proposition 2.1. For integers $s, n, m$ such that $s^{2}+\left(n^{2} \wedge m^{2}\right) \neq 0$, define $C_{s, n, m}$ via the equation

$$
\frac{1}{2 \pi} \int_{-\pi}^{\pi}\left[\pi_{-m}(t)-\pi_{-n}(t)\right] e^{-i s t} d t=\frac{1}{\pi \sigma^{2}} \log \frac{\|[s, n]\|}{\|[s, m]\|}+C_{s, n, m} .
$$

(a) $C_{s, n, m}$ is bounded if and only if $E_{0}\left[\left|S_{1}^{0}\right|^{2} \log \left|S_{1}^{0}\right|\right]<\infty$, and if this is the case $C_{s, n, m}$ tends to zero as both $|s|+|n|$ and $|s|+|m|$ go to $\infty$.

(b) Uniformly in $s, C_{s, n, m}=o(|n-m| /(|n| \wedge|m|))$ as $|n| \wedge|m| \rightarrow \infty$.

(c) Uniformly in $s, C_{s, n, m}=o\left(1 \vee \log \left\{n^{2} /\left(s^{2}+m^{2}\right)\right\}\right)$ as $n /(|m| \vee 1) \rightarrow \infty$.

As an immediate corollary of Proposition 2.1 we obtain

Corollary 2.1. Suppose that $n \rightarrow \infty, s / n \rightarrow \alpha \in \mathbf{R} \cup\{ \pm \infty\}, m / n \rightarrow \beta \in \mathbf{R}$. Then

$$
\int_{-\pi}^{\pi}\left(\pi_{-m}(t)-\pi_{-n}(t)\right) e^{-i s t} d t \longrightarrow \frac{1}{\sigma^{2}} \log \left(\frac{\left(\alpha / \sigma_{1}\right)^{2}+\left(1 / \sigma_{2}\right)^{2}}{\left(\alpha / \sigma_{1}\right)^{2}+\left(\beta / \sigma_{2}\right)^{2}}\right),
$$

where the limit value is understood to be 0 or $\infty$ according as $|\alpha|=\infty$ or $\alpha=\beta=0$. For each $M>1$ the convergence is uniform for $s$ and $m$ such that $|m|<M n$ and $|m| \vee|s|>n / M$. 
Proof of Proposition 2.1. Let $J=J_{s, n, m}$ be the function given by (2.4). Then

$$
C_{s, n, m}=\frac{1}{(2 \pi)^{2}} \int_{-\pi}^{\pi} e^{-i s t} d t \int_{-\pi}^{\pi}\left[\frac{1}{1-\psi(t, l)}-\frac{2}{Q(t, l)}\right]\left(e^{i m l}-e^{i n l}\right) d l+J
$$

According to Lemma $2.1 J \rightarrow 0$ as $|s|+(|n| \wedge|m|) \rightarrow \infty$. The repeated integral on the right side is decomposed into the sum of the integral

$$
\int_{(-\pi, \pi]^{2}}\left[\frac{1}{1-\psi}-\frac{2}{Q}\right]\left(1-e^{-i s t+i n l}\right) d t d l
$$

and a similar one (with $m$ in place of $n$ ). As $|s|+|n| \rightarrow \infty$, the first integral converges to a finite limit (which equals $\int_{(-\pi, \pi]^{2}}[1 /(1-\psi)-2 / Q] d t d l$ ) or diverges to $+\infty$ according as $E_{0}\left[\left|S_{1}^{0}\right|^{2} \log \left|S_{1}^{0}\right|\right]<$ $\infty$ or $=\infty$ (cf. [8]: proof of its Theorem 1), so that if $E_{0}\left[\left|S_{1}^{0}\right|^{2} \log \left|S_{1}^{0}\right|\right]<\infty, C_{s, n, m}$ remains bounded, and otherwise it diverges to $+\infty$ as $|n| \rightarrow \infty$ with $s, m$ being fixed. This completes the proof of (a).

For the proof of (b) we may suppose $|m| \leq n$. Put $k=n-m$. By Lemma 2.1 $J \leq C m^{-2}=o(k / n)$ (as $|m| \rightarrow \infty$ ). We must prove that the double integral in $(2.5)$ is $o(k /|m|)$. To this end we break it into three parts by dividing the inner integral at $l= \pm 1 / n$. The part corresponding to the interval $|l|<1 / n$ is easy to evaluate to be $o(k / n)$. For the evaluation of the remaining parts we put

$$
D(t, l)=\frac{1}{1-\psi(t, l)}-\frac{2}{Q(t, l)} \text { and } \quad K^{ \pm}=\int_{-\pi}^{\pi} e^{-i s t} d t \int_{1 / n}^{\pi} D(t, \pm l)\left(e^{ \pm i m l}-e^{ \pm i n l}\right) d l .
$$

It remains to prove $K^{+}+K^{-}=o(k / m)$. Since the factor $e^{-i s t}$ does not come into play at all, let $s=0$ for simplicity. Writing $e^{i m l}-e^{i n l}=\left(e^{-i k l}-1\right) e^{i n l}$ we integrate by parts to have

$$
\begin{array}{r}
K^{+}=\frac{1}{i n} \int_{-\pi}^{\pi} d t\left[\left(e^{i m l}-e^{i n l}\right) D\right]_{l=1 / n}^{\pi}+\frac{k}{n} \int_{-\pi}^{\pi} d t \int_{1 / n}^{\pi} e^{i m l} D d l \\
-\frac{1}{i n} \int_{-\pi}^{\pi} d t \int_{1 / n}^{\pi}\left(e^{-i k l}-1\right) e^{i n l} \partial_{l} D d l .
\end{array}
$$

The first term on the right side is dominated by $k n^{-2} \int_{0}^{\pi}|D(t, 1 / n)| d t+r=o(k / n)+r$, where $r$ is the boundary term corresponding to $\pi$ and cancels out with that arising from $K^{-}$. Integrating by parts once more and using the relations

$$
\int_{-\pi}^{\pi}\left|\partial_{l}^{v} D(t, l)\right| d t=o\left(l^{-v-1}\right) \quad \text { as } l \rightarrow 0 \quad(v=0,1,2),
$$

one can easily deduce that the second and third terms are $o(k / m)$ and $o(k / n)$, respectively.

We continue the argument made above for the proof of (c). Obviously $K^{ \pm}=o(\log n)$ (uniformly in $s, k)$, hence one may suppose $|m| \wedge|s| \rightarrow \infty$. First consider the case $|m|>|s|$. We must prove that $K^{ \pm}=o(\log (n /|m|))$ as $n /|m| \rightarrow \infty$. The contribution to $K^{ \pm}$of the part involving $e^{i n l}$ tends to zero as $n \rightarrow 0$ uniformly in $s$, namely

$$
\sup _{s}\left|\int_{-\pi}^{\pi} e^{-i s t} d t \int_{1 / n}^{\pi} D(t, \pm l) e^{ \pm i n l} d l\right| \rightarrow 0 \quad \text { as } n \rightarrow \infty
$$


With this in mind we see that as $|m| \rightarrow \infty$

$$
\left|\int_{-\pi}^{\pi} e^{-i s t} d t \int_{1 / n}^{\pi} D(t, l) e^{i m l} d l\right| \leq C \int_{1 / n}^{1 /|m|} d l \int_{0}^{\pi}|D| d t+o(1)=o(1 \vee \log (n /|m|)),
$$

in view of (2.6). Now let $s|s| \geq|m|$. In view of (2.7), with symmetric roles of $t$ and $l$ in $D(t, l)$ taken into account, it suffices to prove that uniformly in $m$,

$$
M^{+}:=\int_{0}^{1 / s} e^{-i s t} d t \int_{1 / n}^{\pi} D(t, l) e^{i m l} d l=o(1 \vee \log (n / s))
$$

as $s \rightarrow \infty$. Changing the variable of integration we see that for any $\varepsilon>0$ and $N>0$

$$
\begin{aligned}
\left|M^{+}\right| \leq \frac{1}{s^{2}} \int_{0}^{1} d t \int_{s / n}^{s \pi} D(t / s, l / s) d l & \leq \int_{0}^{1} d t\left[\int_{s / n}^{N} \frac{\varepsilon}{t^{2}+l^{2}} d l+\int_{N}^{\infty} \frac{C}{l^{2}} d l\right] \\
& \leq 2 \varepsilon\left[(\log (n / s)+\log N]+\frac{C}{N}\right.
\end{aligned}
$$

for all sufficiently large $s$. Thus (2.8) is verified. Proof of Proposition 2.1 is complete.

Proposition 2.2. As $|k| \rightarrow \infty$, uniformly for $n \geq|k|$ and for $s \in \mathbf{Z}$,

$$
\int_{-\pi}^{\pi} \frac{\left(\pi_{0}(t)-\pi_{k}(t)\right) \pi_{-n}(t)}{\pi_{0}(t)} e^{-i s t} d t=\frac{1}{\sigma^{2}} \log \left(\frac{s^{2}+\lambda^{2}(n+|k|)^{2}}{s^{2}+\lambda^{2} n^{2}}\right)+o\left(\frac{k}{n}\right) .
$$

Proof. Write $\rho^{\circ}(t)$ for $1 / \pi_{0}^{\circ}(t)=\sigma^{2}|t|$ and make decomposition

$$
\begin{aligned}
\rho\left[\pi_{0}-\pi_{k}\right] \pi_{-n}= & \rho^{\circ}\left[\pi_{0}^{\circ}-\pi_{k}^{\circ}\right] \pi_{-n}^{\circ}+\left\{\rho\left[\pi_{0}-\pi_{k}\right]-\rho^{\circ}\left[\pi_{0}^{\circ}-\pi_{k}^{\circ}\right]\right\} \pi_{-n}^{\circ} \\
& +\rho\left[\pi_{0}-\pi_{k}\right]\left(\pi_{-n}-\pi_{-n}^{\circ}\right) .
\end{aligned}
$$

On using (2.2) the Fourier transform of the first term equals

$$
\frac{2}{\sigma^{2}} \int_{0}^{\infty} \frac{e^{-\lambda n t}-e^{-\lambda(n+|k|) t}}{t} \cos s t d t=\frac{1}{\sigma^{2}} \log \left(\frac{s^{2}+\lambda^{2}(n+|k|)^{2}}{s^{2}+\lambda^{2} n^{2}}\right),
$$

hence gives the principal term of the formula of the lemma.

For the remaining terms we use certain estimates of Fourier type integrals, which are collected in Appendix (D). From Lemma 6.1 (i) there it follows that

$$
\rho(t)\left|\pi_{0}(t)-\pi_{k}(t)\right| \leq C|k t|,
$$

which implies $\int_{-\pi}^{\pi} \rho\left[\pi_{0}-\pi_{k}\right]\left(\pi_{-n}-\pi_{-n}^{\circ}\right) e^{-i s t} d t=o(k / n)$ in view of Lemma 6.2 (ii).

By Lemma 6.1 (i)

$$
\lim _{k \rightarrow \infty} k^{-1} \sup _{0<|t|<1}\left|\pi_{0}(t)-\pi_{k}(t)-\left[\pi_{0}^{\circ}(t)-\pi_{k}^{\circ}(t)\right]\right|=0 .
$$

This, together with 2.9$)$, shows that the middle term on the right side of $[2.9)$ is $e^{-\lambda n|t|}(|k| \times o(|t|)+$ $|t| \times o(k)$ ), hence its contribution is $o(k / n)$ as above (but this time not only $n$ but $|k|$ must also be made large indefinitely: otherwise $o(k / n)$ must be replaced by $O(k / n)$ ). 
REMARK 6. If $a^{\{d=2\}}([s, n])$ denotes the potential function of the two-dimensional walk $S^{x}$, namely $a^{\{d=2\}}([s, n])=\sum_{j=0}^{\infty}\left(P\left[S_{j}^{0}=0\right]-P\left[S_{j}^{[s, n]}=0\right]\right)$, then

$$
a^{\{d=2\}}([-s, n])-a^{\{d=2\}}([-s, n-k])=\frac{1}{2 \pi} \int_{-\pi}^{\pi}\left[\pi_{-n}(t)-\pi_{-n+k}(t)\right] e^{-i s t} d t .
$$

The asymptotic estimates of $a^{\{d=2\}}([s, n])$ given in [3] or [8] provide better estimates of $C_{s, n, m}$ than in Proposition 2.1 but under certain stronger moment conditions.

\section{Proof of Theorems 1.1 and 1.2}

For simplicity we let $0<|k| \leq n$ unless contrary is stated, which gives rise to no loss of generality as being pointed out in Remark 4. We continue to suppose that $Q$ is diagonal.

Set for $(\alpha, \delta) \in \mathbf{R}^{2}$ with $|\alpha|+|1-\delta|>0$,

$$
\Lambda(\alpha, \delta)=\frac{4 \delta_{+}}{\alpha^{2}+(1-\delta)^{2}} \quad\left(\delta_{+}=(|\delta|+\delta) / 2\right)
$$

write

$$
s_{*}=s / \sigma_{1}, n_{*}=n / \sigma_{2} \text { and } k_{*}=k / \sigma_{2},
$$

and for $|s|+|n-k|>0$, define $r(s, k, n)$ by

$$
G_{L}([0, n],[s, k])=\left(2 \pi \sigma^{2}\right)^{-1} \log \left(1+\Lambda\left(s_{*} / n_{*}, k_{*} / n_{*}\right)\right)+r(s, k, n) .
$$

It follows that $\|[s, n]\|^{2}=\sigma^{2}\left(s_{*}^{2}+n_{*}^{2}\right)$. For simple random walk the reflection principle may apply and it immediately follows from an asymptotic expansion of the potential function $a^{\{d=2\}}$ as found in [3], [5] or [2] that

$$
r(s, k, n)=O\left(\left(s^{2}+|n-k|^{2}+1\right)^{-1}\right) .
$$

We are to find a reasonable estimate of $r$ in general case.

We first consider the case when $n \rightarrow \infty$ under the constraint

$$
\Lambda(s / n, k / n)=\frac{4 n k_{+}}{s^{2}+(n-k)^{2}} \asymp 1,
$$

namely $M^{-1} \leq \Lambda(s / n, k / n) \leq M$ for some $M>0$. This condition is equivalent (or understood to be so) to the condition that there exists a constant $\varepsilon, 0<\varepsilon<1$ such that $k>\varepsilon n$; $|s|<n / \varepsilon$; and either $k<(1-\varepsilon) n$ or $|s|>\varepsilon n$.

Theorem 3.1. As $n \rightarrow \infty$ under (3.1), $r(s, k, n) \rightarrow 0$ uniformly for $s, k$ subject to the restriction specified above (for each $\varepsilon$ ); in particular, if $k_{*} / n_{*} \rightarrow \delta, s_{*} / n_{*} \rightarrow \alpha, 0<\delta \leq 1$ and $|1-\delta|+|\alpha|>0$, then $G_{L}([0, n],[s, k]) \rightarrow\left(2 \pi \sigma^{2}\right)^{-1} \log (1+\Lambda(\alpha, \delta))$.

Proof. The integrand of the integral on the right side of (1.3) (with $\mu=0, n>0$ ) may be written in the form

$$
\pi_{-n+k}(t)-\rho(t) \pi_{k}(t) \pi_{-n}(t)=\pi_{-n+k}(t)-\pi_{-n}(t)+\left[\pi_{0}(t)-\pi_{k}(t)\right] \rho(t) \pi_{-n}(t),
$$


and the assertion is inferred as an immediate consequence of Propositions 2.1 and 2.2 .

Next consider the case when

$$
\Lambda(s / n, k / n) \rightarrow \infty .
$$

Theorem 3.2. As $n \rightarrow \infty$ under (3.3), namely as $k / n \rightarrow 1$ and $s / n \rightarrow 0$, the difference

$$
G_{L}([0, n],[s, k])-\frac{1}{\pi \sigma^{2}} \log \frac{\|[s, n+k]\|}{\|[s, n-k]\| \vee 1}
$$

remains uniformly bounded if and only if $E_{0}\left[\left|S_{1}^{0}\right|^{2} \log \left|S_{1}^{0}\right|\right]<\infty$. If this is the case it converges to zero as $|s|+|n-k| \rightarrow \infty$; if not it diverges to $+\infty$ as $n \rightarrow \infty$ whenever $|s|+|n-k|$ is confined in any finite set.

Proof. Here again the expression on the right side of $(3.2)$ is employed. From Proposition 2.2 it follows that $(2 \pi)^{-1} \int_{-\pi}^{\pi}\left(\pi_{k}-\pi_{0}\right) \rho \pi_{-n} e^{-i s t} d t \rightarrow\left(\pi \sigma^{2}\right)^{-1} \log 2$ in the limit under (3.3). Combined with this as well as with the equality $\lim \|[s, n+k]\| /\|[s, n]\|=2$ (in the same limit) (a) of Proposition 2.1 shows the assertion of Theorem 3.2 .

Proof of Theorem 1.1 On looking at (3.2) the first half of Theorem 1.1 follows from Propositions 2.1 and 2.2. The second half is included in Theorem 3.2 .

Proof of Theorem 1.2 If $|s| \rightarrow \infty$ but $n$ remains in a finite set, then the assertion of Theorem 1.2 is rather trivial since then the probability that the walk $S^{[0, n]}$ visits $[s, k]$ earlier than $L$ tends to zero. If $n \rightarrow \infty$, we have only to apply Propositions 2.1 and 2.2 as in the proof of Theorem 3.1 since $m=n-k=n+|k|$ in the case $k<0$.

\section{Proof of Theorem 1.3}

Theorem 1.3 pertains to the case when $\Lambda(s / n, k / n) \rightarrow 0$ and follows if we prove

Theorem 4.1. Suppose that $E\left[|\tilde{X}|^{2} \log |\tilde{X}|\right]<\infty$. Then, as $(k \vee 1) /(n \vee|s|) \rightarrow 0$

$$
G_{L}([0, n],[s, k])=\frac{1}{\pi} \frac{\sigma_{2}^{2} a^{*}(n) a^{*}(-k)+k_{*} n_{*}}{\|[s, n]\|^{2}}+o\left(\frac{|n k|}{s^{2}+n^{2}}\right) \text { for } n k \neq 0 .
$$

Proof. As in the preceding section suppose that $0<|k| \leq n$ and $Q$ is diagonal. The proof is given in the following three subcases

(i) $|s| \leq n ; k=o(n)$, (ii) $n \leq|s| ; k=o(|s|)$ and (iii) $|s| \leq n ; n \asymp(-k) \vee 1$,

separately. Put

$$
\mathrm{e}_{n}(t)=\pi_{-n}(t)-\pi_{0}(t)+a(n)
$$

and

$$
f(x)=|x|^{-1}\left(e^{-|x|}-1\right)+1\left(=\frac{1}{2 !}|x|-\frac{1}{3 !}|x|^{2}+\cdots\right)
$$

as in [9] (see (5.12) of the next section for how $f$ comes up in the next lemma). We need the following result from [9] (Lemmas 4.2 and 5.5). 
Lemma 4.1. (a) $\rho(t)=\sigma^{2}|t|+o(t), \rho^{\prime}(t)=\sigma^{2} t /|t|+o(1)$ and $\rho^{\prime \prime}(t)=o(1 / t)$ as $t \rightarrow 0$;

(b) $\mathrm{e}_{n}(t)=\sigma_{d}^{-2}|n| f(\lambda n t)+\eta_{n}(t)$ with $\sup _{n}|n|^{-1}\left|\eta_{n}^{(j)}(t)\right|=o\left(|t|^{-j}\right)$ for $j=0,1,2$; in particular, each of $\mathrm{e}_{n}(t) / n, t \mathrm{e}_{n}^{\prime}(t) / n$ and $t^{2} \mathrm{e}_{n}^{\prime \prime}(t) / n$ tends to zero as $n t \rightarrow 0$ and is uniformly bounded. (Here $\eta^{(j)}$ denotes the derivative of the $j$-th order.)

Case (i) $|s| \leq n ;|k|=o(n)$. We make the decomposition

$$
\pi_{-n+k}-\rho \pi_{-n} \pi_{k}=\left(\pi_{-n+k}-\pi_{-n}\right)+a(-k) \rho \pi_{-n}-\rho \pi_{-n} \mathrm{e}_{-k} .
$$

The contribution to $G_{L}$ of the first term on the right side is given in Proposition 2.1 (b) and that of the second is given by Theorem 1.1 of [9] (in view of (1.2)). The sum of these two may be written as

$$
\frac{1}{2 \pi \sigma^{2}} \log \frac{\|[s, n]\|}{\|[s, n-k]\|}+\frac{a(-k) n}{\pi\|[s, n]\|^{2}}+o(k / n)=\frac{a(-k) n+k_{*} n_{*}}{\pi\|[s, n]\|^{2}}+o(k / n)
$$

as $n \rightarrow \infty$ under (i). As for the third term we write

$$
\int_{-\pi}^{\pi}\left(\rho \pi_{-n} \mathrm{e}_{-k}\right)(t) e^{-i s t} d t=\int_{-\pi}^{\pi}\left(\rho \pi_{-n}^{\circ} \mathrm{e}_{-k}\right)(t) e^{-i s t} d t+\int_{-\pi}^{\pi}\left(\rho\left[\pi_{-n}-\pi_{-n}^{\circ}\right] \mathrm{e}_{-k}\right)(t) e^{-i s t} d t .
$$

Having the bound $\left|\left(\rho \pi_{-n}^{\circ} \mathrm{e}_{-k}\right)(t)\right| \leq C[|k t| \wedge 1] e^{-\lambda n|t|}$ in view of 2.2$)$ and Lemma 4.1 (b), we infer that the first integral on the right side is $o(k / n)$. That the second one admits the same estimate follows from Lemma 6.2 with the help of the bound $\left|\mathrm{e}_{-k}(t)\right| \leq C|k|$. Thus we obtain the relation of Theorem 4.1.

Case (ii) $n \leq|s| ;|k|=o(|s|)$. Here we make the decomposition

$$
\begin{aligned}
\pi_{-n+k}-\rho \pi_{-n} \pi_{k}= & \left(\pi_{-n+k}-\pi_{-n}-\pi_{k}+\pi_{0}\right)-\left(\pi_{-n}-\pi_{0}\right)\left(\pi_{k}-\pi_{0}\right) / \pi_{0} \\
= & \left(\pi_{-n+k}-\pi_{-n}-\pi_{k}+\pi_{0}\right)-\rho \mathrm{e}_{n} \mathrm{e}_{-k} \\
& +a(n) \rho \mathrm{e}_{-k}+a(-k) \rho \mathrm{e}_{n}-a(n) a(-k) \rho .
\end{aligned}
$$

It suffices to compute the Fourier inversions for the first two terms of the right-most member, the other terms being dealt with in [9] (see (4.17) and (4.18) given at the end of the present proof). The first and second of the two is dealt with in Lemmas 4.2 and 4.3 below, respectively.

Lemma 4.2. If $E\left[X^{2} \log |X|\right]<\infty$, then as $|s| \rightarrow \infty$

$$
\int_{-\pi}^{\pi}\left(\pi_{-n+k}-\pi_{-n}-\pi_{k}+\pi_{0}\right)(t) e^{-i s t} d t=\frac{1}{\sigma^{2}} \log \left[\frac{\left(s_{*}^{2}+k_{*}^{2}\right)\left(s_{*}^{2}+n_{*}^{2}\right)}{s_{*}^{2}\left(s_{*}^{2}+\left(n_{*}-k_{*}\right)^{2}\right)}\right]+o\left(\frac{n k}{s^{2}}\right) .
$$

Proof. Write

$$
\int_{-\pi}^{\pi}\left(\pi_{-n+k}-\pi_{-n}-\pi_{k}+\pi_{0}\right)(t) e^{-i s t} d t=I+r
$$

with

$$
I=I(s, k, n)=\int_{-\pi}^{\pi} e^{-i s t} w(t) d t \int_{-\pi}^{\pi} \frac{2\left(e^{i(n-k) l}-e^{i n l}-e^{-i k l}+1\right)}{Q(t, l)} d l,
$$




$$
r=r(s, k, n)=\int_{-\pi}^{\pi} e^{-i s t} d t \int_{-\pi}^{\pi}\left[\frac{1}{1-\psi(t, l)}-\frac{2 w(t)}{Q(t, l)}\right]\left(e^{i n l}-1\right)\left(e^{-i k l}-1\right) d l,
$$

where $w(t)$ is a smooth cutoff function such that $w \geq 0$; $w=1$ for $|t|<1 / 2$; $w=0$ for $|t|>1$. As before we have that for any $N$

$$
I=\frac{1}{\sigma^{2}} \log \left[\frac{\left(s_{*}^{2}+k_{*}^{2}\right)\left(s_{*}^{2}+n_{*}^{2}\right)}{s_{*}^{2}\left(s_{*}^{2}+\left(n_{*}-k_{*}\right)^{2}\right)}\right]+O\left(|s|^{-N}\right)
$$

as $|s| \rightarrow \infty$. As for the error term $r$, perform integration by parts twice (to have the factor $s^{-2}$ ) and decompose $\left(e^{i n l}-1\right)\left(e^{-i k l}-1\right)=\sin n l \sin k l+A_{n, k}(l)$, where

$$
A_{n, k}(l)=(\cos n l-1)(\cos k l-1)-i(\cos n l-1) \sin k l+i(\cos k l-1) \sin n l .
$$

Observe

$$
F(t, l):=\partial_{t}^{2}\left[\frac{1}{1-\psi(t, l)}-\frac{2 w(t)}{Q(t, l)}\right]=o\left(\frac{1}{\left(t^{2}+l^{2}\right)^{2}}\right) \quad(|t| \vee|l| \rightarrow 0),
$$

so that $\int_{(-\pi, \pi]^{2}}|F(t, l)| \times d t l^{3} \rightarrow 0$ as $l \rightarrow 0$, and then deduce that

$$
\sup _{k, n}\left|\int_{-\pi}^{\pi} e^{-i s t} d t \int_{-\pi}^{\pi} F(t, l) \frac{A_{n, k}(l)}{n k} d l\right| \rightarrow 0 \quad(|s| \rightarrow \infty)
$$

(cf. Lemma 11.1 of [9]). In order to deal with the contribution of $\sin n l \sin k l$ we need the moment condition $E\left[X^{2} \log |X|\right]<\infty$, with which we proceed as in [9]: Lemma 6.3 (estimation of $\Theta_{I I}$ ). We decompose $F(t, l)=V(t, l)+R(t, l)$, where

$$
V(t, l)=\frac{E\left[X^{2} e^{i Y l}\left(e^{i X t}-1\right)\right]}{(1-\psi(t, l))^{2}}
$$

and $R$ is the rest. On the one hand $R$ admits once more differentiation with $\partial_{t} R=o\left((|t|+|l|)^{-3}\right)$, and the integration by parts shows that its contribution is $o(n k)$. On the other hand $V$ satisfies

$$
\int_{-\pi}^{\pi} \int_{-\pi}^{\pi}\left(t^{2}+l^{2}\right)|V(t, l)| d t d l<\infty
$$

if $E\left[X^{2} \log |X|\right]<\infty$, so that the Riemann-Lebesgue lemma apply. These together show that

$$
\sup _{k, n}\left|\int_{-\pi}^{\pi} e^{-i s t} d t \int_{-\pi}^{\pi} F(t, l) \frac{\sin n l \sin k l}{n k} d l\right| \rightarrow 0 \quad(|s| \rightarrow \infty) .
$$

(Here the outer integral (for the $R$ part) must in general be understood improper and for the integral on $|t|<1 /|s|$ one should integrate by parts back to have the result above.) Consequently $r(s, k, n)=$ $s^{-2} n|k| \times o(1)$ with $o(1) \rightarrow 0$ as $|s| \rightarrow \infty$ uniformly in $n, k$.

The next lemma is subtler than the preceding one.

Lemma 4.3. If $E\left[X^{2} \log |X|\right]<\infty$, then as $|s| \rightarrow \infty$ with $k=o(s)$ and $n=O(s)$

$$
\int_{-\pi}^{\pi} \rho(t) \mathrm{e}_{n}(t) \mathrm{e}_{-k}(t) e^{-i s t} d t=o\left(\frac{n k}{s^{2}}\right) .
$$


Proof. Although one can proceed by extending the lines of the proof of Theorem 1.2 of [9] (given in Sections 3 and 6), where evaluation of the integral $\int \rho \mathrm{e}_{n} e^{-i s t} d t$ is carried out, we proceed somewhat differently in a way the proof works better in the higher dimensions.

From Lemma 4.1 we have the expression $\mathrm{e}_{n}(t)=f_{n}(t)+\eta_{n}(t)$ with the estimates

$$
\left|f_{n}^{(j)}(t)\right| \leq C n(|n t| \wedge 1)|t|^{-j} \text { and } \eta_{n}^{(j)}(t)=n \times o\left(t^{-j}\right) \text { for } j=0,1,2,
$$

where $f_{n}(t)=\sigma_{d}^{-2}|n| f(\lambda n t)$. In addition to the fact that both $\rho$ and $\mathrm{e}_{n}$ do not necessarily admit the differentiation of the third order, the difference of estimates between the derivatives of $f_{n}$ and those of $\eta_{n}$ as given above causes the complication of arguments. To make the proof conceptually clear we replace $\left(\rho \mathrm{e}_{n} \mathrm{e}_{-k}\right)(t)$ by $\sigma^{2}\left(|t| f_{n} f_{-k}\right)(t) w(t)$ and compute the corresponding integral of the latter and that of the difference between the two, separately. First we consider the difference, for which we need to find a way round the lack of differentiability. Write $\rho^{\circ}(t)$ for $\sigma^{2}|t|$ (as in (2.9)) and put

$$
g(t)=\left(\rho \mathrm{e}_{n} \mathrm{e}_{-k}\right)(t)-\left(\rho^{\circ} f_{n} f_{-k}\right)(t) w(t) .
$$

We may suppose that $0<k \leq n \leq s$. After integrating by parts once we split the range of integration at $t= \pm 1 /|s|$. From Lemma 4.1 it follows that under the constraint of the variables $s, n, s$ of the lemma

$$
\sup _{|t|<1 / s}\left|\left(\rho \mathrm{e}_{n} \mathrm{e}_{-k}\right)^{\prime}(t)\right| / n k \rightarrow 0 \quad \text { as } s \rightarrow \infty
$$

which entails the same relation for $g^{\prime}$ in place of $\left(\rho \mathrm{e}_{n} \mathrm{e}_{-k}\right)^{\prime}$. We then deduce that the Fourier integral of $g$ on $|t|<1 /|s|$ is $o\left(k n / s^{2}\right)$, and one more integration by parts gives

$$
s^{2} \int_{-\pi}^{\pi} g(t) e^{-i s t} d t=-\int_{1 /|s|<|t|<\pi} g^{\prime \prime}(t) e^{-i s t} d t+o(n k) .
$$

For the latter we express $g$ as the telescopic sum $\left(\rho-\rho^{\circ}\right) f_{n} f_{k}+\left(\mathrm{e}_{n}-f_{n}\right) \rho f_{k}+\rho \mathrm{e}_{n}\left(\mathrm{e}_{-k}-f_{k}\right)$ and deal with them separately. Among them we consider only the last term and verify that

$$
\int_{1 /|s|<|t|<\pi}\left(\rho \mathrm{e}_{n} \eta_{-k}\right)^{\prime \prime}(t) e^{-i s t} d t=o(n k)
$$

the integrals for the other two being evaluated in the same way in view of (4.7).

For the proof of (4.10) we claim that

$$
\int_{1 /|s|<|t|<\pi}\left[\rho \mathrm{e}_{n}\right](t) \eta_{-k}^{\prime \prime}(t) e^{-i s t} d t=o(n k) .
$$

Since $\left|\rho \mathrm{e}_{n}\right| \leq C|n t|$ and $\eta_{-k}^{\prime \prime}(t)=k \times o\left(t^{-2}\right)$, the integrand is $n k \times o(1 / t)$. Let $F$ and $V$ be the functions given in (4.5) and (4.6), respectively. We may write

$$
\eta_{-k}^{\prime \prime}(t)=\frac{1}{2 \pi} \int_{-\pi}^{\pi} F(t, l)\left(1-e^{-i k l}\right) d l+r_{k}(t)
$$

with $r_{n}(t)$, a nice function that is negligible for the present purpose, and then $\eta_{-k}^{\prime \prime}(t)=v_{k}(t)+\tau_{k}(t)$, where

$$
v_{k}(t)=\frac{1}{2 \pi} \int_{-\pi}^{\pi} V(t, l)\left(1-e^{-i k l}\right) d l
$$


and $\tau_{k}$ is the rest. Then, as in the preceding proof, we see that the function $\tau_{k}(t)$ is differentiable for $t \neq 0, \tau_{k}^{\prime}(t) / k=o\left(1 / t^{3}\right)$ and its contribution is $o(n k)$ and that if $E\left[X^{2} \log |X|\right]<\infty$, the RiemannLebesgue lemma yields

$$
\int_{1 /|s|<|t|<\pi}\left[\rho \mathrm{e}_{n}\right](t) v_{k}(t) e^{-i s t} d t=o(n k)
$$

That the last estimate is uniform in $n$ and $k$ requires proof. Since, by dominated convergence, $\int_{-\pi}^{\pi}\left|t v_{k}(t)\right| d t / k \rightarrow 0$ as $|k| \rightarrow \infty$, it suffices for the proof to show that $n^{-1}$ times the integral restricted on $|t|>\varepsilon$ tends to zero uniformly in $n$ for each $\varepsilon>0$ and $k$. This follows from the fact that $\mathrm{e}_{n}=\pi_{-n}-\pi_{0}+a(n)$ and $\sup _{\varepsilon<|t|<\pi}\left|\pi_{-n}(t)-\pi_{0}(t)\right|=o(n)$. Thus the claim (4.11) has been verified. For the proof of 4.10 we must evaluate the integrals of other terms of $\left(\rho \mathrm{e}_{n}\left[\mathrm{e}_{-k}-f_{k}\right]\right)^{\prime \prime}(t)$, e.g., $\rho^{\prime \prime}(t) \mathrm{e}_{n}(t)\left[\mathrm{e}_{-k}-f_{k}\right](t)$, but their evaluations are quite similar, hence omitted.

It remains to prove

$$
\int_{\mathbf{R}}\left(\rho^{\circ} f_{n} f_{k}\right)(t) w(t) e^{-i s t} d t=o\left(\frac{n k}{s^{2}}\right) .
$$

It is not hard at all to verify this as in a similar way to the above, but we take up another way. We are concerned with the Fourier integral that has an explicit form if $w$ is removed and we shall seek out it. To this end the following decomposition of $\rho^{\circ} f_{n} f_{k}$ is convenient:

$$
\begin{aligned}
\rho^{\circ} f_{n} f_{k}= & \rho^{\circ}\left(\pi_{n}^{\circ}-\pi_{0}^{\circ}\right)\left(\pi_{k}^{\circ}-\pi_{0}^{\circ}\right) \\
& +\sigma_{2}^{-2} k \rho^{\circ}\left(\pi_{n}^{\circ}-\pi_{0}^{\circ}\right)+\sigma_{2}^{-2} n \rho^{\circ}\left(\pi_{k}^{\circ}-\pi_{0}^{\circ}\right)+\sigma_{2}^{-4} n k \rho^{\circ} .
\end{aligned}
$$

Remember the identity $\pi_{n}(t)=e^{-\lambda|n t|} / \sigma^{2}|t|$ given in $(2.2)$ and observe that $\rho^{\circ} \pi_{n}^{\circ} \pi_{k}^{\circ}=\pi_{n+k}^{\circ}$, so that the first term on the right side of (4.14) equals $\pi_{n+k}^{\circ}-\pi_{n}^{\circ}-\pi_{k}^{\circ}+\pi_{0}^{\circ}$, whose Fourier transform, already computed in the preceding lemma, equals

$$
\frac{1}{\sigma^{2}} \log \left[\frac{\left(s_{*}^{2}+k_{*}^{2}\right)\left(s_{*}^{2}+n_{*}^{2}\right)}{s_{*}^{2}\left(s_{*}^{2}+\left(n_{*}+k_{*}\right)^{2}\right)}\right]=\frac{-n k}{\sigma_{2}^{2} \pi\|[s, n]\|^{2}}+o\left(\frac{n k}{s^{2}}\right) .
$$

The other terms are the transforms of Cauchy densities and by inverting them we have

$$
\frac{n k}{\sigma_{2}^{2} \pi\|[s, n]\|^{2}}+\frac{n k}{\sigma_{2}^{2} \pi\|[s, k]\|^{2}}-\frac{n k}{\sigma_{2}^{2} \pi\|[s, 0]\|^{2}}
$$

for the Fourier integral of their sum (but the last term necessitates truncation by $w$ or, otherwise, interpretation as a Schwartz distribution). Now summing (4.15) and (4.16) gives (4.13). This completes the proof of Lemma 4.3 .

Now we can finish the proof of Theorem 4.1 in the case (ii). Proposition 6.1 of [9] says that if $E\left[X^{2} \log |X|\right]<\infty$, then as $|s| \rightarrow \infty$ with $n \leq|s|$

$$
\frac{a^{*}(-k)}{2 \pi} \int_{-\pi}^{\pi} \rho(t) \mathrm{e}_{n}(t) e^{-i s t} d t=\frac{a^{*}(-k) n}{\pi \sigma^{2}}\left[\frac{1}{s_{*}^{2}+n_{*}^{2}}-\frac{1}{s_{*}^{2}}\right]+o\left(\frac{n k}{s^{2}}\right)
$$

and the corresponding Fourier coefficient of $a^{*}(n) \rho(t) \mathrm{e}_{-k}(t)$ is $O\left(n k^{3} / s^{4}\right)$, hence $o\left(n k / s^{2}\right)$; from [9] we also have

$$
\frac{a^{*}(-k) a^{*}(n)}{2 \pi} \int_{-\pi}^{\pi}(-\rho(t)) e^{-i s t} d t=\frac{a^{*}(-k) a^{*}(n) \sigma_{2}^{2}}{\pi \sigma^{2} s_{*}^{2}}+o\left(\frac{n k}{s^{2}}\right) .
$$


Observe that under the constraint of (ii), $(2 \pi)^{-1}$ times the right side of the formula in Lemma 4.2 may be written as $n_{*} k_{*} / \pi\|[s, n]\|^{2}+o\left(n k / s^{2}\right)$ and that the sum of (4.17) and (4.18) may be written as $\sigma_{2}^{2} a^{*}(n) a^{*}(-k) / \pi\|[s, n]\|^{2}+o\left(n k / s^{2}\right)$ since $\lim a(n) / n=1 / \sigma_{2}^{2}$. Combined with (4.3) and Lemma 4.3 , these observations yield the estimate of Theorem 4.1.

Case (iii) $|s| \leq n ;(-k) \vee 1 \asymp n$. It suffices to prove that $G_{L}([0, n],[s, k])=o(1)$ uniformly for $|s| \leq$

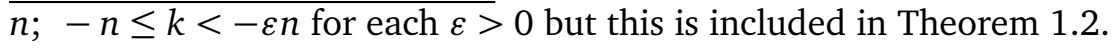

The proof of Theorem 4.1 , hence that of Theorem 1.3 is complete.

\section{The Walks in Dimensions $d \geq 3$}

This section consists of five subsections and some preliminary discussions given preceding them. Here our primary purpose is to prove Theorems 1.4 and 1.5, of which the proof of the latter is given in Subsection 1. After obtaining some preliminary estimates in Subsections 3 and 4, we derive in Subsection 4 an estimate of the hitting distribution $H_{[0, n]}$ (Theorem 5.1) under the same moment condition of Theorem 1.4, and, succeeding it, we prove Theorem 1.4. In [9] (Theorem 10.1) the present author has presented the same asymptotic form of $H_{[0, n]}$ as in Theorem 5.1 and erroneously asserted that it holds without any additional moment condition (i.e., under the existence of second moments) for $d \geq 3$. We shall see in the last subsection that how $H_{0}(x)=G_{L}([0, n],[x, 0])$ may behave when $E|X|^{d}=\infty$, which in particular shows that the formula for $H_{[0, n]}$ as given in Theorem 5.1 cannot be assured under the second moment condition only.

Let the random walk $S_{n}^{0}$ on $\mathbf{Z}^{d}$ be irreducible and has zero mean and finite variances. Throughout this section the dimension $d$ is supposed to be greater than or equal to three. For $x \in \mathbf{Z}^{d-1}$ and $n \in \mathbf{Z}$ we denote by $[x, n]$ the $d$-dimensional point $\left(x_{1}, \ldots, x_{n-1}, n\right) \in \mathbf{Z}^{d}$ and by $L$ the hyper plane $\left\{[x, 0]: x \in \mathbf{Z}^{d-1}\right\}$. The random variables $X, Y$, the norm $\|[x, n]\|$, the Green function $G_{L}$, the functions $\rho, \pi_{n}$ etc. are all understood to be analogously defined. The Euclidian norm is denoted by $|\cdot|$ and we often write $\theta^{2}$ for $|\theta|^{2}, \theta \in \mathbf{R}^{d-1}$.

An obvious analogue of the Fourier representation formula (1.3) (as well as that of (1.2) ) is valid in the dimensions $d \geq 3$. The leading term in the asymptotic formula of Theorem 1.4 comes from the explicit expression of the Fourier integral that intrinsically arises when $1-\psi$ is replaced by $\frac{1}{2} Q$ in the representation formula of $G_{L}$. The problem is to estimate the error term that is caused by the replacement; for the estimation we need some moment condition, of which the condition (1.5) (resp. $\left.E|X|^{d}<\infty\right)$ is appropriate under the constraint $|x|<M(|n| \vee|k|)$ (resp. $\left.|x| \geq M^{-1}|n| \vee|k|\right)$.

Throughout the rest of this section we suppose that $X$ and $Y$ are uncorrelated so that

$$
Q(\theta, l)=R(\theta)+\sigma_{d}^{2} l^{2} \quad \text { where } R(\theta):=E\left[(X \cdot \theta)^{2}\right] \text { and } \sigma_{d}^{2}=E\left[Y^{2}\right] .
$$

Let $n>0$, write $s=|x|$ and define $\pi_{-n}^{\circ}(\theta)$ analogously to 2.1 . It follows that $\pi_{0}^{\circ}(\theta)=1 / \sqrt{\sigma_{d}^{2} R(\theta)}$. Hence, putting $|\theta|_{R}=\sqrt{R(\theta) / \operatorname{det} R}, \lambda=\sigma^{d} / \sigma_{d}^{2}=\sqrt{\operatorname{det} R} / \sigma_{d}$ and $\rho^{\circ}(\theta)=1 / \pi_{0}^{\circ}(\theta)$ we have

$$
\rho^{\circ}(\theta)=\sqrt{\sigma_{d}^{2} R(\theta)}=\sigma^{d}|\theta|_{R}
$$

and

$$
\pi_{-n}^{\circ}(\theta)=\frac{1}{2 \pi} \int_{\mathbf{R}} \frac{2}{Q(\theta, l)} e^{i n l} d l=\frac{e^{-n \lambda|\theta|_{R}}}{\sigma^{d}|\theta|_{R}} .
$$


Let $T^{\prime}=[-\pi, \pi)^{d-1}$ be the range of the variable $\theta ; w(\theta)$ denotes a smooth cut off function as before ( $w$ equals 1 about the origin and vanishes for $|\theta|>1$ ). For every $N>0$, as $s \rightarrow \infty$

$$
\frac{\sigma^{d}}{(2 \pi)^{d-1}} \int_{T^{\prime}}|\theta|_{R} w(\theta) e^{-i x \cdot \theta} d \theta=\frac{\Gamma(d / 2) \sigma_{d}^{2}}{\pi^{d / 2}\|[x, 0]\|^{d}}+o\left(s^{-N}\right) .
$$

Indeed, together with the change of variables of integration, Green's formula transforms the integral above into $(d-2) \sigma_{d}^{2} \sigma^{-d+2}\|[x, 0]\|^{-2} \int_{\mathrm{R}^{d-1}}|\theta|^{-1} e^{i x^{\prime} \cdot \theta} d \theta+o\left(s^{-N}\right)$, where $x^{\prime}=R^{-1 / 2} x$ (cf. [8]:Lemma 2.1), and a known explicit formula for the last integral ([6], p.73) yields (5.2).

With these preliminaries Theorems 1.4 and 1.5 are proved independently except for the case when $k / n \rightarrow-1$ (see the end of Subsection 4).

\subsection{Proof of Theorem 1.5}

Lemma 5.1. Let $|k| \leq n$ and $D_{x, n, k}$ be defined via the equation

$$
\frac{1}{(2 \pi)^{d-1}} \int_{T^{\prime}} \frac{\left[\pi_{0}(\theta)-\pi_{k}(\theta)\right] \pi_{-n}(\theta)}{\pi_{0}(\theta)} e^{-i x \cdot \theta} d \theta=\frac{\kappa_{d}}{\sigma^{2}}\left[\frac{1}{\|[x, n]\|^{d-2}}-\frac{1}{\|[x,|k|+n]\|^{d-2}}\right]+D_{s, n, k} .
$$

Then, under (1.6), $\sup _{k}\left|D_{x, n, k}\right|=o\left(1 / n^{d-2}\right)$ as $n \rightarrow \infty$ and under (1.5), $\sup _{n} n^{d-1}\left|D_{x, n, m}\right|=o(|k|)$ as $|k| \rightarrow \infty$, where the supremums are taken under the constraint $|k| \leq n$ and both formulae are valid uniformly in $|x|<M n$ for each $M>1$.

(If $\mu \neq 0,\|[x,|k|+n]\|$ appearing in the right side is to be replaced by $\|[x+2 n \mu, k+n]\|$ for $k>0$ and by $\|[x, k-n]\|$ for $k<0$. ; also $\|[x, n]\|$ to be replaced by $\|[x,-n]\|=\|[x+2 n \mu, n]\|)$

Proof. The proof is similar to that of Proposition 2.2. We have the same decomposition of $\rho\left[\pi_{0}-\right.$ $\left.\pi_{k}\right] \pi_{-n}$ as in (2.9) and the same estimate for each terms of it but with $|\theta|$ in place of $|t|$. Thus

$$
\left(\rho^{\circ}\left[\pi_{0}^{\circ}-\pi_{k}^{\circ}\right] \pi_{-n}^{\circ}\right)(\theta)=\frac{e^{-n \lambda|\theta|_{R}}-e^{-(n+|k|) \lambda|\theta|_{R}}}{\sigma^{d}|\theta|_{R}}
$$

for the first term, whose Fourier coefficient (i.e. integral on $T^{\prime}$ ) therefore agrees with the principal term of the formula of the lemma up to $o\left(n^{-N}\right)$ for any $N$. The middle term is of the form $e^{-\lambda n|\theta|}(|k| \times o(|\theta|)+|\theta| \times o(k))$ and it is readily inferred that its Fourier coefficientl is $o\left(k / n^{d-1}\right)$ under $(1.6)$. For the last term, which is $\rho\left[\pi_{0}-\pi_{k}\right]\left(\pi_{-n}-\pi_{-n}^{\circ}\right)$, we first observe that

$$
\left(\rho\left|\pi_{0}-\pi_{k}\right|\right)(\theta) \leq C[|k \theta| \wedge 1]
$$

(see Lemma 6.1 (i) for the case $|k \theta|<1$ ) and then infer from (i) and (ii) of Lemma 6.2 that its contribution is $o\left(n^{-d+2}\right)$ under (1.6) and $o\left(k / n^{d-1}\right)$ under $(1.5)$, respectively.

Proof of Theorem 1.5. Under (1.6) it holds that for each $M>1$, uniformly for $|x|<M n$, as $n \rightarrow \infty$

$$
G([n, 0],[x, 0])=\frac{1}{(2 \pi)^{d-1}} \int_{T^{\prime}} \pi_{-n}(\theta) e^{-i x \cdot \theta} d \theta=\frac{\kappa_{d}}{\sigma^{2}\|[x, n]\|^{d-2}}(1+o(1))
$$

(see [8] for the identification of the leading term and Lemma 6.2 for the error estimate). Combining this with Lemma 5.1 (its first case) we find the formula of Theorem 1.5 .

The following lemma that corresponds to (b) of Proposition 2.1 will be applied in the proof of Theorem 1.4. 
Lemma 5.2. If (1.5) holds, $s^{2}+\left(n^{2} \wedge m^{2}\right) \neq 0$ and $C_{x, n, m}$ is defined via the equation

$$
\frac{1}{(2 \pi)^{d-1}} \int_{T^{\prime}}\left[\pi_{-m}(\theta)-\pi_{-n}(\theta)\right] e^{-i x \cdot \theta} d \theta=\frac{\kappa_{d}}{\sigma^{2}}\left[\frac{1}{\|[x, m]\|^{d-2}}-\frac{1}{\|[x, n]\|^{d-2}}\right]+C_{s, n, m},
$$

then, uniformly in $x, C_{x, n, m}=o\left(|n-m| /(|n| \wedge|m|)^{d-1}\right)$ as $|n| \wedge|m| \rightarrow \infty$.

Proof. We have an analogue of (2.5). By making a suitable truncation argument by means of $w(l)$ the term $J$ in it is evaluated to be negligible. As for the double integral in it, writing $e^{i m l}-e^{i n l}=$ $\left(e^{-i k l}-1\right) e^{i n l}(k=n-m)$, we first integrate its inner integral by parts $d-2$ times successively, and then proceed as in the proof of (b) of Proposition 2.1 (with the help of Lemma 6.2 of Appendix (D)) to obtain the required estimate.

Remark 7. The case $|n / m| \rightarrow \infty$ is excluded in Lemma 5.2, since in its subcase $|m| / s \rightarrow 0$ we need to impose an additional moment condition on $X$ for identifying the asymptotic form of $G([0, m],[0, x])=(2 \pi)^{-d+1} \int_{T^{\prime}} \pi_{-m}(\theta) e^{-i x \cdot \theta} d \theta$ (cf. [8] $)$.

\subsection{Estimation of $\rho(\theta)-\rho^{\circ}(\theta)$}

We infer that

$$
\pi_{0}(\theta)-\pi_{0}^{\circ}(\theta)=o\left(|\theta|^{-1}\right)
$$

Hence,

$$
\rho(\theta)-\sigma^{d}|\theta|_{R}=\rho(\theta)-\rho^{\circ}(\theta)=o(|\theta|) .
$$

We denote by $\nabla$ the gradient operator w.r.t. $\theta$ and write

$$
s=|x|, \omega=x / s \text { and } \nabla_{\omega}=\omega \cdot \nabla .
$$

Lemma 5.3. Suppose $E|X|^{d}<\infty$. Then for $k=0,1,2, \ldots, d$,

$$
\nabla_{\omega}^{k}\left(\rho-\rho^{\circ}\right)(\theta)=o\left(|\theta|^{-k+1}\right)
$$

and

$$
\nabla_{\omega}^{d}\left(\rho-\rho^{\circ}\right)(\theta)=O\left(|\theta|^{-1}\right)+\tau(\theta)
$$

with some differentiable function $\tau(\theta)$ on $T^{\prime} \backslash\{0\}$ such that $\nabla \tau(\theta)=o\left(|\theta|^{-d}\right)$.

Proof. As in the proof of Proposition 2.1 let $D=D(\theta, l)$ denote the difference of $(1-\psi)^{-1}$ and 2/Q:

$$
D(\theta, l)=\frac{1}{1-\psi(\theta, l)}-\frac{2}{Q(\theta, l)}=\frac{\psi(\theta)-1+\frac{1}{2} Q(\theta, l)}{(1-\psi(\theta, l)) \frac{1}{2} Q(\theta, l)} .
$$

Putting $U=\rho-\rho^{\circ}$ and recalling that $\pi_{0}^{\circ}=1 / \rho^{\circ}$ we have

$$
\nabla U(\theta)=-\rho^{2}(\theta) \nabla\left[\pi_{0}-\pi_{0}^{\circ}\right](\theta)+U(\theta)\left(\rho(\theta)+\rho^{\circ}(\theta)\right) \nabla \pi_{0}^{\circ}(\theta) .
$$

Since $\nabla D(\theta, l)=o\left((|\theta|+|l|)^{-3}\right)$ as $|\theta|+|l| \rightarrow 0$, we have $\nabla\left[\pi_{0}-\pi_{0}^{\circ}\right](\theta)=\frac{1}{2 \pi} \int_{-\pi}^{\pi} \nabla D d l+O(|\theta|)=$ $o\left(|\theta|^{-2}\right)$, which, together with (5.4), gives the desired bound for $k \leq 1$. In general for $1 \leq k \leq d$,

$$
\nabla_{\omega}^{k} D(\theta, l)=o\left((|\theta|+|l|)^{-k-2}\right)
$$


hence $\nabla_{\omega}^{k}\left[\pi_{0}-\pi_{0}^{\circ}\right](\theta)=o\left(|\theta|^{-k-1}\right)$. Further noting that $\nabla_{\omega}^{k} \rho(\theta)=O\left(|\theta|^{-k+1}\right)$ we obtain the first bound of the lemma for $k \geq 2$. The second one is obtained by defining $\tau(\theta)$ via the equation

$$
-\rho^{2}(\theta) \nabla_{\omega}^{d}\left[\pi_{0}-\pi_{0}^{\circ}\right](\theta)=-\rho^{2}(\theta) \frac{i^{d}}{2 \pi} \int_{-\pi}^{\pi} \frac{E\left[e^{i X \cdot \theta+i Y l}(X \cdot \omega)^{d}\right]}{(1-\psi(\theta, l))^{2}} d l+\tau(\theta) .
$$

Proposition 5.1. Suppose $E|X|^{d}<\infty$. Then $H_{0}(x)=\frac{\Gamma(d / 2) \sigma_{d}^{2}}{\pi^{d / 2}\|[x, 0]\|^{d}}+o\left(s^{-d}\right)$.

Proof. Using the bound (5.5) we readily deduce that

$$
H_{0}(x)=\frac{1}{(2 \pi)^{d-1}} \int_{T^{\prime}} \rho^{\circ}(\theta) w(\theta) e^{-i x \cdot \theta} d \theta+\frac{i^{d}}{(2 \pi)^{d-1} s^{d}} \int_{T_{1 / s}^{\prime}} \nabla_{\omega}^{d}\left(\rho-\rho^{\circ}\right)(\theta) e^{-i x \cdot \theta} d \theta+o\left(s^{-d}\right),
$$

where $T_{\varepsilon}^{\prime}=\left\{\theta \in T^{\prime}:|\theta|>\varepsilon\right\}(\varepsilon>0)$. Now substitute from (5.5) and apply the Riemann-Lebesgue lemma to see that the second integral above takes on the form $o(1)+\int_{T_{1 / s}^{\prime}} \tau(\theta) e^{-i x \cdot \theta} d \theta$, of which, on integrating by parts once more, the integral term is also $o(1)$. Finally the formula (5.2) concludes that of the lemma.

\subsection{Estimation of $\mathrm{e}_{n}(\theta)$}

Remember that $f(x)=|x|^{-1}\left(e^{-|x|}-1\right)+1$ and $\mathrm{e}_{n}(\theta)=\pi_{-n}(\theta)-\pi_{0}(\theta)+a(n)$ (Section 4).

Lemma 5.4. Suppose that $E|X|^{d}<\infty$. Then $\mathrm{e}_{n}(\theta)=\sigma_{d}^{-2}|n| f\left(\lambda n|\theta|_{R}\right)+\eta_{n}(\theta)$ with

$$
\sup _{n, \omega} \frac{1}{|n|}\left|\nabla_{\omega}^{k} \eta_{n}(\theta)\right|=o\left(|\theta|^{-k}\right) \quad \text { for } \quad k=0,1, \ldots, d
$$

and

$$
\sup _{n, \omega} \frac{1}{|n|}\left|\nabla_{\omega}^{d} \eta_{n}(\theta)-\tau_{n}(\theta)\right|=O\left(|\theta|^{-2}\right)
$$

for some differentiable function $\tau_{n}(\theta)$ on $T^{\prime} \backslash\{0\}$ such that $\sup _{n}\left|n^{-1} \nabla \tau_{n}(\theta)\right|=o\left(|\theta|^{-d-1}\right)$.

Proof. Put $W(\theta, l)=\left[\frac{1}{1-\psi(\theta, l)}-\frac{1}{1-\phi(l)}\right]-\left[\frac{2}{Q \theta, l)}-\frac{2}{\sigma_{d}^{2} l^{2}}\right]$, which may also be written as

$$
W(\theta, l)=\frac{\psi-\phi}{(1-\psi)(1-\phi)}-\frac{-2 R(\theta)}{Q(\theta, l) \sigma_{d}^{2} l^{2}} .
$$

The function $f$ comes up from the corresponding integral of the second term:

$$
\begin{aligned}
\frac{1}{2 \pi} \int_{-\pi}^{\pi} \frac{-2 R(\theta)}{Q(\theta, l) \sigma_{d}^{2} l^{2}}(\cos n l-1) d l & =\frac{1}{\pi \sigma_{d}^{2}} \int_{0}^{n} d u \int_{0}^{\pi} \frac{2 R(\theta)}{Q(\theta, l)} \cdot \frac{\sin u l}{l} d l \\
& =\sigma_{d}^{-2}|n| f\left(\lambda n|\theta|_{R}\right)+O\left(\theta^{2}\right),
\end{aligned}
$$

where the formula $a^{2} \int_{0}^{\infty}\left[\pi\left(a^{2}+l^{2}\right) l\right]^{-1} \sin y l d l=\frac{1}{2}\left(1-e^{-|a| y}\right)$ (valid for $\left.y \geq 0\right)$ is used for the last equality. It accordingly follows that $2 \pi \eta^{n}(\theta)=\int_{-\pi}^{\pi} W(\theta, l)\left(e^{i n l}-1\right) d l+$ a negligible term. The 
evaluation of the last integral is made in the same way as for the two dimensional case for here the integration by $\theta$ is not involved. This gives (5.9). Since $\nabla W=\nabla D$, we have for $k \geq 1$,

$$
\nabla_{\omega}^{k} \eta_{n}(\theta)=\frac{1}{2 \pi} \int_{-\pi}^{\pi} \nabla_{\omega}^{k} D(\theta, l)\left(e^{i n l}-1\right) d l
$$

and the formula $(5.10)$ is obtained as in the proof of Lemma 5.3 .

Remark 8. Suppose $E\left[\left[\left.X\right|^{d}+|Y|^{d}\right]<\infty\right.$. Then $\psi-1+\frac{1}{2} Q=O\left(|\theta|^{3}+|l|^{3}\right)$, from which we infer that for $|\theta|<1 / 2, \pi_{0}(\theta)=1 / \sigma^{d}|\theta|_{R}+O\left(\log |\theta|^{-1}\right)$, hence $\rho(\theta)-\rho^{\circ}(\theta)=O\left(\theta^{2} \log |\theta|^{-1}\right)$; moreover the estimates of Lemmas 5.3 and 5.4 are improved as follows: for $k=0, \ldots, d$,

$$
\nabla_{\omega}^{k}\left(\rho-\rho^{\circ}\right)(\theta)=O\left(|\theta|^{-k+2} \log |\theta|^{-1}\right) \text { and } \sup _{n, \omega}|n|^{-1}\left|\nabla_{\omega}^{k} \eta_{n}(\theta)\right|=O\left(|\theta|^{-k+1} \log |\theta|^{-1}\right),
$$

respectively, and the use of these considerably simplifies the argumenys in the next section.

\subsection{Estimation of $H_{[0, n]}(x)$ and Proof of Theorem 1.4}

As has been noticed in the two-dimensional case Theorem 1.4 includes, as a special case of $k=0$, the asymptotic estimate of the hitting distribution $H_{[0, n]}(x)$. For the proof of Theorem 1.4 this special case is essential and first dealt with.

Theorem 5.1. (i) Suppose (1.5) to hold. Then uniformly for $|x|<M|n|$, as $|n| \rightarrow \infty$

$$
H_{[0, n]}(x)=\frac{\Gamma(d / 2)}{\pi^{d / 2}} \cdot \frac{|n|}{\|[x,-n]\|^{d}}(1+o(1)) .
$$

(ii) Suppose that $E|X|^{d}<\infty$. Then uniformly for $|n|<M|x|$, as $|x| \rightarrow \infty$

$$
H_{[0, n]}(x)=\frac{\Gamma(d / 2)}{\pi^{d / 2}}\left(\frac{\sigma_{d}^{2} a^{*}(n)}{\|[x, 0]\|^{d}}+\frac{|n|}{\|[x,-n]\|^{d}}-\frac{|n|}{\|[x, 0]\|^{d}}\right)+o\left(\frac{n}{s^{d}}\right) .
$$

Proof. First we prove (i). Let $n>0$. The leading term should be given by

$$
\frac{1}{(2 \pi)^{d-1}} \int_{\mathbf{R}^{d-1}} \rho^{\circ}(\theta) \pi_{-n}^{\circ}(\theta) e^{-i x \cdot \theta} d \theta=\frac{\Gamma(d / 2)}{\pi^{d / 2}} \cdot \frac{n}{\|[x, n]\|^{d}} .
$$

(see Section 10 of [9]). Our task is to prove that uniformly for $|x|<M n$,

$$
\int_{T^{\prime}}\left[\rho(\theta) \pi_{-n}(\theta)-\rho^{\circ}(\theta) \pi_{-n}^{\circ}(\theta) w(\theta)\right] e^{-i x \cdot \theta} d \theta=o\left(n^{-d+1}\right) .
$$

Now suppose (1.5) to hold. We decompose $\rho \pi_{-n}-\rho^{\circ} \pi_{-n}^{\circ}$ as follows:

$$
\rho \pi_{-n}-\rho^{\circ} \pi_{-n}^{\circ}=\rho\left[\pi_{-n}-\pi_{-n}^{\circ}\right]+\left[\rho-\rho^{\circ}\right] \pi_{-n}^{\circ} .
$$

In view of 5.1) (i.e., $\left.\pi_{-n}^{\circ}(\theta)=e^{-n \lambda|\theta|_{R}} / \rho^{\circ}(\theta)\right)$ and the estimate $\rho(\theta)-\rho^{\circ}(\theta)=o(|\theta|$ ) we have

$$
\sup _{x}\left|\int_{T^{\prime}}\left(\rho(\theta)-\rho^{\circ}(\theta)\right) \pi_{-n}^{\circ}(\theta) e^{-i x \cdot \theta} d \theta\right|=o\left(n^{-d+1)}\right) .
$$


We shall see in Appendix (D) (Lemma 6.2 (ii)) that the Fourier coefficient of $\rho\left[\pi_{-n}-\pi_{-n}^{\circ}\right]$ admits the same estimate as above. Thus the proof the part (i) is complete.

Proof of (ii). Remember that $\rho \pi_{n}=\rho \mathrm{e}_{n}+1-a^{*}(n) \rho$. Owing to Theorem 5.1 it therefore suffices to prove

Proposition 5.2. If $E|X|^{d}<\infty$, then uniformly for $|n|<|x|$, as $s=|x| \rightarrow \infty$,

$$
\frac{1}{(2 \pi)^{d-1}} \int_{T^{\prime}} \rho(\theta) \mathrm{e}_{n}(\theta) e^{-i x \cdot \theta} d \theta=\frac{\Gamma(d / 2)}{\pi^{d / 2}}\left(\frac{|n|}{\|[x,-n]\|^{d}}-\frac{|n|}{\|[x, 0]\|^{d}}\right)+o\left(\frac{n}{s^{d}}\right) .
$$

Proof. Recalling that $f(u)=|u|^{-1}\left(e^{-|u|}-1\right)+1$ we put

$$
f_{n}(\theta)=\sigma_{d}^{-2}|n| f\left(n \lambda|\theta|_{R}\right)
$$

Let $n>0$. Then

$$
\sigma^{d}|\theta|_{R} f_{n}(\theta)=e^{-n \lambda|\theta|_{R}}-1+n \lambda|\theta|_{R}
$$

and by Lemma 5.4

$$
\mathrm{e}_{n}(\theta)=f_{n}(\theta)+\eta_{n}(\theta)
$$

with $\nabla_{\omega}^{k} \eta_{n}(\theta)=n \times O\left(|\theta|^{-k+1}\right)$ for $k=0,1, \ldots, d$. Taking this into account we decompose

$$
\left(\rho \mathrm{e}_{n}\right)(\theta)=\left(\rho^{\circ} f_{n}\right)(\theta)+\left(\rho \eta_{n}\right)(\theta)+\left(\left[\rho-\rho^{\circ}\right] f_{n}\right)(\theta) .
$$

Since $\rho \mathrm{e}_{n}$ is periodic, $\int_{T^{\prime}}\left(\rho \mathrm{e}_{n}\right)(\theta)(1-w(\theta)) e^{-i x \cdot \theta} d \theta=o\left(s^{-d}\right)$. On using 5.2

$$
\frac{1}{(2 \pi)^{d-1}} \int_{T^{\prime}}\left(\rho^{\circ} f_{n}\right)(\theta) w(\theta) e^{-i x \cdot \theta} d \theta=\frac{\Gamma(d / 2)}{\pi^{d / 2}}\left(\frac{n}{\|[x,-n]\|^{d}}-\frac{n}{\|[x, 0]\|^{d}}\right)+o\left(\frac{n}{s^{d}}\right) .
$$

It suffices for the proof of Proposition 5.2 to verify that uniformly in $n$,

$$
\frac{s^{d}}{n} \int_{T^{\prime}}\left(\rho \eta_{n} w\right)(\theta) e^{-i x \cdot \theta} d \theta+\frac{s^{d}}{n} \int_{T^{\prime}}\left(\left[\rho-\rho^{\circ}\right] f_{n} w\right)(\theta) e^{-i x \cdot \theta} d \theta=o(1) .
$$

Denote the first and second terms on the left side by $I_{1}$ and $I_{2}$, respectively.

Evaluation of $I_{1}$ is made in a similar way to the proof of Proposition 5.1. We employ Lemma 5.4. After integrating by parts $d-1$ times we split the range of integration along the $(d-2)$-dimensional sphere of radius $1 / s$. The integral inside the sphere is easy to evaluate. For the integral on its outside we integrate by parts once more. The typical term that then arises is a constant multiple of

$$
\frac{1}{n} \int_{T_{1 / s}^{\prime}}\left(w \nabla_{\omega}^{j} \rho \nabla_{\omega}^{d-j} \eta_{n}\right)(\theta) e^{-i x \cdot \theta} d \theta=\int_{T_{1 / s}^{\prime}} o\left(\frac{1}{|\theta|^{j-1}} \times \frac{1}{|\theta|^{d-j}}\right) e^{-i x \cdot \theta} d \theta \quad(0 \leq j \leq d),
$$

plus the boundary term, which is $o(1)$. For $1 \leq j<d$, further performing integration by parts once we see that the last integral is also $o(1)$. For the case $j=0$ we use the second formula of Lemmas 5.4 to obtain the same estimate. As for the case $j=d$, separating the non-differentiable term from $\nabla_{\omega}^{d} \rho$ according to (5.6), we have only to make the same argument. Thus we have $I_{1}=o(1)$ as $s \rightarrow \infty$. Here, the Riemann-Lebesgue lemma is applied to the parts involving the functions $(O(\cdot)$ terms in 
(5.6) and (5.10) ) that are integrable but not necessarily differentiable. That the convergence is uniform in $n \leq s / M$ for such parts is verified as in the relevant discussion following (4.12) in the proof of Lemma 4.3 .

Now we turn to $I_{2}$. We need to derive a bound of $\nabla_{\omega}^{j} f_{n}(\theta)$. First observe that

$$
f^{(j)}(u)=O\left(1 \wedge|u|^{-j-1}\right)
$$

and $\nabla f_{n}(\theta)=\sigma_{d}^{-2} \sigma^{-d} f^{\prime}\left(n \lambda|\theta|_{R}\right) n^{2} R \theta /|\theta|_{R}$. Then one deduces that

$$
\nabla_{\omega}^{j} f_{n}(\theta)=O\left(\left[|n \theta|^{2} \wedge 1\right] \times|\theta|^{-j-1}\right) .
$$

Combining this with the estimate of $\nabla_{\omega}^{d-j}\left(\rho-\rho^{\circ}\right)$ given in Lemma 5.3 one readily obtains that $I_{2}=o(1)$.

Proof of Theorem 1.4 We follow the lines of the proof of Theorem 1.3. While much of details are simplified, there are a few places where modifications are necessitated, which we indicate below.

Case (i) $s \leq n ; k=o(n)$. Here we use the moment condition (1.5) but does not the condition $E|X|^{d}<\infty$. The first step of getting the leading term (see $(4.2)$ ) is cleared by Proposition 5.1 and Lemma 5.2, The rest is the same.

Case (ii) $n \leq s ; k=o(s)$. Here we need only the condition $E|X|^{d}<\infty$. In view of Propositions 5.1 and 5.2 it suffices to obtain the propositions corresponding to Lemmas 4.2 and 4.3 . For the former one, if $F$ is given by $(4.5)$ with $\partial_{t}^{2}$ replaced by $\nabla_{\omega}^{d}$, then $F=o\left((|\theta|+|l|)^{-d-2}\right)$, and we can proceed similarly to the arguments made after it in the proof of Lemma 4.2, except that the leading term is provided by the formula of Lemma 5.2. To prove the analogue of Lemma 4.3 we also follow its proof. For the evaluation of the Fourier coefficient of $g$ (given in (4.8)) we perform integration by parts, successively $d-1$ times, for the integral to be evaluated and then proceed in the same way with the help of Lemmas 5.3 and 5.4. As for the function $\rho^{\circ} f_{k} f_{n}$ treated in the last step of the proof of Lemma 4.3 the explicit forms of the relevant Fourier transforms are derived from the formulae (5.2) and (5.15) and that of Lemma 5.2.

Case (iii) $s \leq n ;(-k) \vee 1 \asymp n$. The assertion follows from Theorem 1.5 (under (1.6)).

5.5. An Estimate of $H_{0}(x)$ under $E\left[|X|^{d}\right]=\infty$

Theorem 5.2. Let $d=3$. Then for each $\varepsilon>0$, as $s:=|x| \rightarrow \infty$

$$
H_{0}(x)=\frac{\sigma_{3}^{2}}{2 \pi\|[x, 0]\|^{3}}+\frac{1}{s^{2}} M_{\varepsilon}(x)+o\left(\frac{\log s}{s^{3}}\right),
$$

where

$$
M_{\varepsilon}(x)=\frac{1}{(2 \pi)^{3}} \int_{T^{\prime}} \rho^{2}(\theta) d \theta \int_{-\pi}^{\pi} \frac{E\left[(\omega \cdot X)^{2} e^{i(X-x) \cdot \theta+i l Y} ;|X-x|<\varepsilon s\right]}{(1-\psi(\theta, l))^{2}} d l .
$$

If $E\left[X^{2} \log |X|\right]<\infty$, the error term in (5.17) can be replaced by $o\left(s^{-3}\right)$.

It is inferred from Theorem 5.2 that for any increasing and unbounded function $h(t), t \geq 0$ and any positive number $\delta$ there exists a probability $p$ on $\mathbf{Z}^{3}$ of zero mean such that $E\left[|X|^{2+\delta}\right]<\infty, Y$ is a.s. 
bounded and $H_{0}(x)|x|^{3-\delta} h(|x|)$ is unbounded. Similar results are obtained for $d>3$ although here we do not discuss on them.

Proof of Theorem 5.2. The proof starts from the expression

$$
[2 \pi s]^{2} H_{0}(s)=-s^{2} \int_{T^{\prime}} \rho(\theta) e^{-i x \cdot \theta} d \theta=\int_{T^{\prime}} \nabla_{\omega}^{2} \rho(\theta) e^{-i x \cdot \theta} d \theta .
$$

Differentiate both sides of (5.7) and observe that $-\rho^{2} \nabla_{\omega}^{2}\left(\pi_{0}-\pi_{0}^{\circ}\right)=\Lambda_{0}+\Lambda_{1}$, where

$$
\Lambda_{0}(\theta)=-\frac{\rho^{2}(\theta)}{2 \pi}\left[\int_{-\pi}^{\pi} \frac{E\left[e^{i X \cdot \theta+i l Y}(X \cdot \omega)^{2}\right]}{[1-\psi(\theta, l)]^{2}} d l+\int_{\mathbf{R}} \frac{-E\left[(X \cdot \omega)^{2}\right]}{\left[\frac{1}{2} Q(\theta, l)\right]^{2}} d l\right]
$$

and $\Lambda_{1}$ is the rest, for which $\Lambda_{1}=o\left(1 /|\theta|^{3}\right)$ and $\nabla \Lambda_{1}=o\left(1 /|\theta|^{4}\right)$. Then we have

$$
\nabla_{\omega}^{2} \rho(\theta)=\nabla_{\omega}^{2} \rho^{\circ}(\theta)+\Lambda_{0}+\Lambda_{1}+\Lambda_{2}
$$

where

$$
\Lambda_{2}=-\nabla_{\omega}\left[\rho^{2}\right](\theta) \nabla_{\omega}\left[\pi_{0}-\pi_{0}^{\circ}\right](\theta)+\nabla_{\omega}\left(U\left[\rho+\rho^{\circ}\right] \nabla_{\omega} \pi_{0}^{\circ}\right)(\theta) .
$$

We do not make truncation by means of $w(\theta)$ at this stage; the boundary terms that arise in the integration by parts (of non-periodic functions) that will be performed once more cancel out one another since $\nabla_{\omega}^{2} \rho$ is periodic. By $(5.2)$ we obtain that

$$
\int_{T^{\prime}} \nabla_{\omega}^{2} \rho^{\circ}(\theta) e^{-i x \cdot \theta} d \theta=[2 \pi s]^{2} \times \frac{\sigma_{d}^{2}}{2 \pi\|[x, 0]\|^{3}}+o\left(s^{-1}\right)+\text { the boundary term } .
$$

For both $\Lambda_{1}$ and $\Lambda_{2}$ we have a situation similar to one arising in the proofs of Lemmas 6.2 and 6.3 of [9] (but only with the appearance here more complicated) and by virtually the same arguments as are made therein it is shown that $\int_{T^{\prime}} \Lambda_{j}(\theta) e^{-x \cdot \theta} d \theta=o\left(s^{-1} \log s\right)$ for $j=1$, 2 , where the error term is replaced by $o\left(s^{-1}\right)$ plus the boundary term if $E\left[X^{2} \log |X|\right]<\infty$.

It remains to evaluate the Fourier coefficients of $\Lambda_{0}$. It is easy to see that

$$
\int_{T^{\prime}} \Lambda_{0}(\theta) e^{-i x \cdot \theta} d \theta=\int_{T_{1 / s}^{\prime}} \zeta(\theta) e^{-i x \cdot \theta} d \theta+o(1 / s)
$$

where

$$
\zeta(\theta)=\frac{[\rho(\theta)]^{2}}{2 \pi} \int_{-\pi}^{\pi} \frac{E\left[\left(e^{i X \cdot \theta}-1\right) e^{i Y l}(\omega \cdot X)^{2}\right]}{[1-\psi(\theta, l)]^{2}} d l .
$$

Note that $\zeta$ may not admit differentiation and that it is periodic. In what follows we prove that

$$
J(x):=\int_{T_{1 / s}^{\prime}} \zeta(\theta) e^{-i x \cdot \theta} d \theta=(2 \pi)^{2} M_{\varepsilon}(\theta)+o(1) .
$$

We decompose $\zeta=\zeta_{0}+\zeta_{1}+\zeta_{2}$, where, by writing $X_{w}=X \cdot \omega$,

$$
\zeta_{0}(\theta)=\frac{\rho^{2}}{2 \pi} \int_{-\pi}^{\pi} \frac{E\left[X_{\omega}^{2} e^{i l Y} e^{i X \cdot \theta}(1-w(|X| \theta))\right]}{(1-\psi)^{2}} d l,
$$




$$
\zeta_{1}(\theta)=-\frac{\rho^{2}}{2 \pi} \int_{-\pi}^{\pi} \frac{E\left[X_{\omega}^{2} e^{i l Y}(1-w(|X| \theta))\right]}{(1-\psi)^{2}} d l
$$

and

$$
\zeta_{2}(\theta)=\frac{\rho^{2}}{2 \pi} \int_{-\pi}^{\pi} \frac{E\left[X_{\omega}^{2} e^{i l Y}\left(e^{i X \cdot \theta}-1\right) w(|X| \theta)\right]}{(1-\psi)^{2}} d l
$$

We may suppose that $w(u)=1$ for $|u|<\frac{1}{4}$ and 0 for $|\theta|>\frac{1}{2}$. Note that $\mid \nabla_{\omega}^{j} w(|X| \theta)(|X||\theta|)^{j}$ is bounded by a constant times the indicator function $1\left(\frac{1}{4}<|X||\theta|<\frac{1}{2}\right)$.

On observing that $\nabla_{\omega}^{j} \zeta_{1}(\theta)=o\left(|\theta|^{-j-1}\right)$ for $j=0,1,2$ integrations by parts give

$$
\int_{T_{1 / s}^{\prime}} \zeta_{1}(\theta) e^{-i x \cdot \theta} d \theta=\frac{i}{s} \int_{\partial T^{\prime}} \zeta_{1}(\theta) e^{-i x \cdot \theta} \omega \cdot n d \sigma+o(1 / s),
$$

where $n$ denotes the outer normal vector to $\partial T^{\prime}$. The boundary integral above vanishes since if $X \neq 0, w(|X| \theta)=0$ on $\partial T^{\prime}$ so that the integrand is periodic.

On integrating by parts and applying Fubini's theorem

$$
\begin{aligned}
\int_{T_{1 / s}^{\prime}} \zeta_{2}(\theta) e^{-i x \cdot \theta} d \theta= & E\left[\int_{T_{1 / s}^{\prime}} \frac{e^{-i x \cdot \theta} d \theta}{2 \pi s} \int_{-\pi}^{\pi} \frac{\rho^{2} X_{\omega}^{3} e^{i l Y} e^{i X \cdot \theta} w(|X| \theta)}{(1-\psi)^{2}} d l\right] \\
& +\int_{T_{1 / s}^{\prime}} \frac{e^{-i x \cdot \theta} d \theta}{2 \pi s i} \int_{-\pi}^{\pi} E\left[X_{\omega}^{2} e^{i l Y}\left(e^{i X \cdot \theta}-1\right) \nabla_{\omega}\left(\frac{\rho^{2} w(|X| \theta)}{(1-\psi)^{2}}\right)\right] d l .
\end{aligned}
$$

The first term on the right side is $o(1 / s)$. Indeed if the expectation above is restricted to the event $|X|<K$, it is $o(1 / s)$ for each $K$ in view of the Riemann-Lebesgue lemma. Changing the variables of the outer integral we see that the same expectation but on $|X| \geq K$ is bounded in absolute value by a positive multiple of

$$
E\left[\int_{\mathbf{R}^{2}} \frac{u^{2} w(u) d u}{2 \pi s} \int_{\mathbf{R}} \frac{\left|X_{\omega}\right|^{3}}{|X|\left(u^{2}+l^{2}\right)^{2}} d l ;|X| \geq K\right] \leq \frac{1}{s} E\left[X^{2}:|X|>K\right] .
$$

The second term is similarly dealt with. If the expectation involved in it is restricted to $|X| \leq K$, the corresponding part is $o(1 / s)$. The other part, after integrating by parts once more, is disposed of in the same way. These together verify

$$
\int_{T_{1 / s}^{\prime}} \zeta_{2}(\theta) e^{-i x \cdot \theta} d \theta=o(1 / s)
$$

We are left with $\zeta_{0}$. Let $0<\varepsilon<1 / 2$. Split the range of integration into two parts according as $|X-x| \geq \varepsilon s$ or $|X-x|<\varepsilon s$ and call $J_{1}$ and $J_{2}$, respectively, their contributions to $\int_{T_{1 / s}^{\prime}} \zeta_{0}(\theta) e^{-i x \cdot \theta} d \theta$. Since $J_{2}=(2 \pi)^{2} M_{\varepsilon}(x)$ if $\varepsilon<1 / 2$, it suffices to show that $J_{1}=o(1)$. We integrate by parts with respect to $\theta$ by factorizing the integrand as $e^{i(X-x) \cdot \theta} \times\left(\right.$ the other) to deduce that for each $\varepsilon>0, J_{1}$ equals

$$
-\int_{T_{1 / s}^{\prime}} d \theta \int_{-\pi}^{\pi} E\left[\frac{X_{\omega}^{2} e^{i(X-x) \cdot \theta}}{2 \pi i|X-x|} e^{i l Y} \nabla_{\omega}\left(\frac{(1-w(|X| \theta)) \rho^{2}}{(1-\psi)^{2}}\right) ;|X-x| \geq \varepsilon s\right] d l
$$


plus the boundary integral that is $o(1 / s)$ (the integral on $\partial T^{\prime}$ vanish by the same reason as before). Repeat the same integration by parts once more and note that both $\nabla w(|X| \theta)$ and $1-w(|X| \theta)$ vanishes if $|X||\theta|<\frac{1}{4}$. We then observe that the double integral above is bounded in absolute value by a constant multiple of

$$
\begin{aligned}
& E\left[\frac{s X_{\omega}^{2}}{|X-x|^{2}} ;|X-x| \geq \varepsilon s,|X| \geq \frac{s}{4}\right]+\int_{1 / s}^{\pi} E\left[\frac{X_{\omega}^{2}}{|X-x|^{2}} ;|X-x| \geq \varepsilon s,|X| \geq \frac{1}{4 r}\right] \frac{d r}{r^{2}} \\
& =o(1 / s) .
\end{aligned}
$$

Finally, observing that the expectation involved in the second term is $s^{-2} \times o(1)$ as $r \rightarrow 0$, we conclude that $J_{1}=o(1 / s)$ as $s \rightarrow \infty$. The proof of (5.18) is complete.

\section{Appendices}

(A) We prove the formula (1.3). The proof is based on the identity

$$
p_{L}^{m}([0, n],[s, k])=p^{m}([0, n],[s, k])-\sum_{\tau=1}^{m} \sum_{j \in \mathbf{Z}} F_{n}(\tau, j) p^{m-\tau}([j, 0],[s, k]) \quad(m \geq 1),
$$

where $p^{m}(x, y)=P\left[S_{m}^{x}=y\right]$ and for $\tau=0,1,2, \ldots$

$$
F_{n}(\tau, s)=P\left[S_{\tau}^{[0, n]}=[s, 0], S_{j}^{[0, n]} \notin L \text { for } j=1, \ldots, \tau-1\right]
$$

(the joint distribution of the time $\tau$ and position $s$ of the first entrance into $L$ of the walk $S^{[0, n]}$ ). It is readily inferred (cf. [9], Appendix A) that for $0<|t| \leq \pi$,

$$
\sum_{m=0}^{\infty} \sum_{s \in \mathbf{Z}} p^{m}(0,[s, k]) e^{i s t}=\pi_{k}(t) \quad \text { and } \sum_{\tau=0}^{\infty} \sum_{j \in \mathbf{Z}} F_{n}(\tau, j) e^{i j t}=\rho(t) \pi_{-n}(t)
$$

and with the help of these identities we derive from (6.1)

$$
\sum_{m=0}^{\infty} \sum_{s \in \mathbf{Z}} p_{L}^{m}([0, n],[s, k]) e^{i s t}=\pi_{-n+k}(t)-\rho(t) \pi_{-n}(t) \pi_{k}(t)
$$

The last double series is absolutely convergent: in fact $\sum_{m=0}^{\infty} \sum_{s \in \mathrm{Z}} p_{L}^{m}([0, n],[s, k])$ is nothing but the Green function of one-dimensional walk killed at the origin and hence equals $a(n)+a(-k)-$ $a(n-k)<\infty$ (cf. [7]). Now the Fourier inversion yields (1.3).

(B) Let $B_{t}$ be the two dimensional standard Brownian motion and consider its linear transform $X_{t}=Q^{1 / 2} B_{t}$. If $g^{\circ}(x, y)$ denotes the Green function of $X_{t}$ killed on $L$ and $g_{B}(x, y)=-\frac{1}{\pi} \log |y-x|$ and $g_{B}^{\circ}(x, y)=g_{B}(x, y)-g_{B}\left(x^{\prime}, y\right)$, where $x^{\prime}$ stands for the mirror image of $x$ relative to the line $Q^{-1 / 2} L$, then, $g^{\circ}(x, y)=g_{B}^{\circ}(A x, A y) \operatorname{det} A(x \in \mathbf{R}, y>0)$ where $A=Q^{-1 / 2}$. We may write $(A x)^{\prime}=A \tilde{x}$, so that

$$
g^{\circ}(x, y)=\frac{1}{2 \pi \sigma^{2}} \log \frac{Q^{-1}(y-\tilde{x})}{Q^{-1}(y-x)} \quad(x \in \mathbf{R}, y>0) .
$$


The point $\tilde{x}$ is characterized by $\tilde{x}_{2}=-x_{2}$ and $A(x-\tilde{x}) \cdot A[1,0]=0$, which is solved to yield $\tilde{x}_{1}=x_{1}-2 \mu x_{2}\left(\mu=\sigma_{12} / \sigma^{2}\right)$. Thus if $x=[0, n]$, then $\tilde{x}=[-2 \mu n,-n]$, and the formula above may be written as

$$
g^{\circ}([0, n],[s, k])=\frac{1}{\pi \sigma^{2}} \log \frac{\|[s+2 \mu n, k+n]\|}{\|[s, k-n]\|} \quad(n k>0) .
$$

in accordance with (6.2) given below.

(C) Here we indicate a way the proofs given in Section 2 are modified in the case $\sigma_{12} \neq 0$. (For those in Sections 3 and 4 see Section 10 of [9].) It is noted that the formula of Theorem 1.1 may be written as

$$
G_{L}([0, n],[s, k])=\frac{1}{\pi \sigma^{2}} \log \frac{\left\|\left[s+2 \mu\left(n+k_{-}\right), n+|k|\right]\right\|}{\|[s, k-n]\| \vee 1}+B_{s, n, k},
$$

where $k_{-}=(|k|-k) / 2$. If we define

$$
\tilde{\pi}_{k}(t)=\frac{1}{2 \pi} \int_{-\pi+\mu t}^{\pi+\mu t} \frac{e^{-i k l}}{1-\psi(t, l-\mu t)} d l,
$$

then

$$
G_{L}([0, n],[s, k])=\frac{1}{2 \pi} \int_{-\pi}^{\pi}\left[\tilde{\pi}_{-n+k}(t)-\rho(t) \tilde{\pi}_{-n}(t) \tilde{\pi}_{k}(t)\right] e^{-i(s+\mu n-\mu k) t} d t .
$$

The principal part of $1-\psi(t, l-\mu t)=1-E\left[e^{i t \tilde{X}+i l Y}\right]$ is given by half the quadratic form

$$
\tilde{Q}(\theta)=Q\left(\theta_{1}, \theta_{2}+\mu \theta_{1}\right)=\tilde{\sigma}_{1}^{2} \theta_{1}^{2}+\sigma_{2}^{2} \theta_{2}^{2} \quad \text { with } \quad \tilde{\sigma}_{1}^{2}=\sigma_{1}^{2}-\mu \sigma_{12}=\sigma^{4} / \sigma_{2}^{2} .
$$

For the quadratic form of the inverse matrix $\tilde{Q}^{-1}$ we have $\tilde{Q}^{-1}(\alpha-\mu \beta, \pm \beta)=Q^{-1}(\alpha, \beta)$; we shall be interested in the following version of it

$$
\begin{aligned}
& \tilde{Q}^{-1}(\alpha+\mu(1-\delta), 1+|\delta|) \\
& =Q^{-1}\left(\alpha+2 \mu\left(1+\delta_{-}\right), 1+|\delta|\right)= \begin{cases}Q^{-1}(\alpha+2 \mu, 1+\delta) & \delta>0, \\
Q^{-1}(\alpha, \delta-1) & \delta<0 .\end{cases}
\end{aligned}
$$

By changing the variables according to $u=l+\mu t$,

$$
\int_{-\pi}^{\pi}\left(\pi_{-m}(t)-\pi_{-n}(t)\right) e^{-i s t} d t=\frac{1}{2 \pi} \int_{-\pi}^{\pi} d t \int_{-\pi+\mu t}^{\pi+\mu t} \frac{A+B}{1-\psi(t, u-\mu t)} d u,
$$

where

$$
A=e^{-i(s+\mu m) t}\left(e^{i m u}-e^{i n u}\right), \quad B=\left(e^{-i(s+\mu m) t}-e^{-i(s+\mu n) t}\right) e^{i n u} .
$$

Denote by $I_{A}$ and $I_{B}$ the integrals corresponding to $A$ and $B$, respectively. Then, the same proofs of Propositions 2.1 and 2.2 yield that in the limit indicated in Corollary 2.1

$$
I_{A} \rightarrow \frac{1}{\sigma^{2}} \log \left(\frac{\tilde{Q}^{-1}(\alpha+\mu \beta, 1)}{\tilde{Q}^{-1}(\alpha+\mu \beta, \beta)}\right) \quad \text { and } \quad I_{B} \rightarrow \frac{1}{\sigma^{2}} \log \left(\frac{\tilde{Q}^{-1}(\alpha+\mu, 1)}{\tilde{Q}^{-1}(\alpha+\mu \beta, 1)}\right)
$$


respectively. This shows Proposition 2.1 that is so modified that the limit in Corollary 2.1 becomes $\sigma^{-2} \log \left[\tilde{Q}^{-1}(\alpha+\mu, 1) / \tilde{Q}^{-1}(\alpha+\mu \beta, \beta)\right]$. For Proposition 2.2 we can proceed similarly. We write down

$$
\int_{-\pi}^{\pi} \frac{\left(\pi_{0}-\pi_{k}\right) \pi_{-n}}{\pi_{0}} e^{-i s t} d t=\frac{1}{(2 \pi)^{2}} \int_{-\pi}^{\pi} d t \int_{-\pi+\mu t}^{\pi+\mu t} \frac{(A+B) d l}{1-\psi(t, l-\mu t)} \int_{-\pi+\mu t}^{\pi+\mu t} \frac{\rho(t) e^{i n u} d u}{1-\psi(t, u-\mu t)},
$$

where

$$
A=e^{-i(s+\mu n) t}\left(1-e^{-i k l}\right), \quad B=\left(e^{-i(s+\mu n)) t}-e^{-i(s+\mu(n-k) t}\right) e^{-i k l},
$$

and denote by $I_{A}$ and $I_{B}$ the corresponding integrals. Then, as $s / n \rightarrow \alpha$ and $k / n \rightarrow \delta$,

$$
I_{A} \rightarrow \frac{1}{\sigma^{2}} \log \left(\frac{\tilde{Q}^{-1}(\alpha+\mu, 1+|\delta|)}{\tilde{Q}^{-1}(\alpha+\mu, 1)}\right), \quad I_{B} \rightarrow \frac{1}{\sigma^{2}} \log \left(\frac{\tilde{Q}^{-1}(\alpha+\mu(1-\delta), 1+|\delta|)}{\tilde{Q}^{-1}(\alpha+\mu, 1+|\delta|)}\right),
$$

which shows Proposition 2.2 modified in an obvious way.

(D) Let $\theta$ denote the $(d-1)$-dimensional variable as in Section 5 (but here the case $d=2$ is included). It is supposed that $X$ and $Y$ are uncorrelated.

Lemma 6.1. (i) There exists a constant $C$ such that $\left|\pi_{0}(\theta)-\pi_{k}(\theta)\right| \leq C|k|(0<|\theta|<1)$.

(ii) $\lim _{k \rightarrow \infty} k^{-1} \sup _{0<|\theta|<1}\left|\pi_{0}(\theta)-\pi_{k}(\theta)-\left[\pi_{0}^{\circ}(\theta)-\pi_{k}^{\circ}(\theta)\right]\right|=0$.

Proof. We decompose $2 \pi\left[\pi_{0}(\theta)-\pi_{k}(\theta)\right]=H_{c}(\theta)+H_{s}(\theta)$, where

$$
H_{c}(\theta)=\int_{-\pi}^{\pi} \frac{1-\cos k l}{1-\psi(\theta, l)} d l \text { and } H_{s}(\theta)=-i \int_{-\pi}^{\pi} \frac{\sin k l}{1-\psi(\theta, l)} d l
$$

We claim that

$$
\frac{1}{k} \sup _{0<|\theta|<1}\left|H_{s}(\theta)\right| \rightarrow 0 \quad \text { as }|k| \rightarrow \infty .
$$

Noticing that only the odd part of $(1-\psi)^{-1}$ is relevant, for the proof we write $\psi(\theta, l)-\psi(\theta,-l)=$ $2 i E\left[\left(e^{i X \cdot \theta}-1\right) \sin Y l\right]+2 i E[\sin Y l]$ so that

$$
\frac{H_{s}(\theta)}{k}=\int_{-\pi}^{\pi} \frac{E\left[\left(e^{i X \cdot \theta}-1\right) \sin Y l\right]}{(1-\psi(\theta, l))(1-\psi(\theta,-l))} \frac{\sin k l}{k} d l+\int_{-\pi}^{\pi} \frac{E[\sin Y l-Y l]}{(1-\psi(\theta, l))(1-\psi(\theta,-l))} \frac{\sin k l}{k} d l .
$$

The first integral is $o(1)$ uniformly in $|\theta|>0$ since its integrand is $o\left(|\theta| /\left[\theta^{2}+l^{2}\right]\right)$ as $|\theta|+|l| \rightarrow 0$ (due to $E[Y X]=0$ ). The integrand of the second one is dominated in absolute value by $C E \mid \sin Y l-$ $\left.Y l|/| l\right|^{3}$, which is integrable so that the dominated convergence theorem may be applied to conclude that it also is $o(1)$. This verifies the claim (6.4).

Since $\int_{-\pi}^{\pi}(1-\cos k l) l^{-2} d l<k \pi$, we have $\left|H_{c}\right| \leq C k$. Combined with $(6.4)$, this shows (i). (ii) is obtained in a similar way.

Let $w(l)$ be a smooth cut-off function as in (4.4). 
Lemma 6.2. Let $h(\theta)$ be a periodic bounded Borel function on the $(d-1)$-dimensional torus $T^{\prime}$ and put

$$
I_{n}=\int_{T^{\prime}} d \theta \int_{-\pi}^{\pi} h(\theta)\left[\frac{1}{1-\psi(\theta, l)}-\frac{2 w(l)}{Q(\theta, l)}\right] e^{i n l} d l .
$$

(i) Let $|h(\theta)| \leq 1$. Then $I_{n}=o\left(n^{-d+2}\right)$, provided that

$$
E\left[Y^{2} \log |Y|\right]<\infty \text { for } d=2,4 \text { and } E\left[|Y|^{d-2}\right]<\infty \text { for } d \geq 5 \text {. }
$$

(ii) Let $|h(\theta)| \leq|\theta|$. Then $I_{n}=o\left(n^{-d+1}\right)$, provided that

$$
E\left[Y^{2} \log |Y|\right]<\infty \text { for } d=3 \text { and } E\left[|Y|^{d-2}\right]<\infty \text { for } d \geq 4 \text {. }
$$

In both (i) and (ii) the estimates of $I_{n}$ hold uniformly for $h(\theta)$ that satisfy the assumed bounds.

Proof. Write $r=\sqrt{\theta^{2}+l^{2}}, B_{n}=\{r \leq 1 / n\}$ and $T=T^{\prime} \times(-\pi, \pi]$. Put

$$
D^{w}(\theta, l)=\frac{1}{1-\psi(\theta, l)}-\frac{2 w(l)}{Q(\theta, l)} .
$$

We prove (ii) first. If $d=2$, we decompose the range $T$ into $B_{n}$ and its complement. Clearly the integral on $B_{n}$ is $o(1 / n)$ under the assumption of (ii). We integrate by parts (w.r.t. the variable $l$ ) the integral on $T \backslash B_{n}$, which is then transformed into

$$
\frac{\text { the boundary integral }}{\text { in }}-\frac{1}{i n} \int_{T \backslash B_{n}} h(\theta) \partial_{l} D^{w}(\theta, l) e^{i n l} d \theta d l .
$$

Since $\left|\partial B_{n}\right|=O(1 / n)$ and $h \partial_{l} D^{w}=o(n)$ on $\partial B_{n}$, the boundary integral is $o(1)$. Integrating the last integral by parts once more we find it to be at most a constant multiple of $n^{-1} \int_{1 / n}^{\infty} r^{-2} d r=o(1)$. Thus $I_{n}=o\left(n^{-1}\right)$ as required.

For $d=3$ we first perform integration by parts to obtain $n I_{n}=i \int_{T} h(\theta) \partial_{l} D^{w}(\theta, l) e^{i n l} d \theta d l$ and then apply the same procedure as above to the right side to see that

$$
n^{2} I_{n}=-\int_{T \backslash B_{n}} h(\theta) \partial_{l}^{2} D^{w}(\theta, l) e^{i n l} d \theta d l+o(1)
$$

We make decomposition $h \partial_{l}^{2} D^{w}=K+\tau$, where

$$
K(\theta, l)=\frac{h(\theta) E\left[e^{i X \cdot \theta}\left(e^{i Y l}-1\right) Y^{2}\right]}{(1-\psi(\theta, l))^{2}},
$$

and $\tau=\tau(\theta, l)$ is the rest. Since then $\tau$ admits one more differentiation w.r.t. $l$ and $\partial_{l} \tau=o\left(1 / r^{3}\right)$, its contribution to the integral in (6.6) is shown to be $o(1)$. Now use the supposed moment condition $E\left[Y^{2} \log |Y|\right]<\infty$ to see that $K$ is integrable on $T$ (cf. Lemma 6.1 of [9]), which fact shows that this integral tends to zero as $n \rightarrow \infty$ in view of the Riemann-Lebesgue lemma.

If $d \geq 4$, under $E\left[|Y|^{d-1}\right]<\infty$ we can decompose $\partial_{l}^{d-1} D^{w}(\theta, l)=v(\theta, l)+\tau(\theta, l)$ with $|v| \leq C r^{-4}$ (so that $h v$ is integrable) and $\left|\partial_{l} \tau\right|=o\left(r^{-d-2}\right.$ ) (due to $E[Y X]=0$ ). As above we then have 
$n^{d-1} I_{n}=i^{d-1} \int_{T^{\prime} \backslash B_{n}} h(\theta)[v+\tau](\theta, l) e^{-i x \cdot \theta+i n l} d \theta d l+o(1)$. The contribution of $v$ is evaluated by the Riemann-Lebesgue lemma and that of $\tau$ by the integration by parts, both resulting in $o(1)$.

The proof of (i) is carried out similarly. For $d=4$ we apply integration by parts two times, which results in $o\left(r^{-4}\right)$ for the integrand, and, the further integration by parts being not allowed under the condition $E Y^{2}<\infty$, we impose the logarithmic moment condition on $Y$ to guarantee the integrability. In the case $d=2$ we also need to suppose the same moment condition of $Y$ to guarantee the integrability of $D^{w}$ (so that $I_{n}=o(1)$ ), but the reason is slightly different: if $d=2$ we cannot dispose of the integral about the origin in advance, $D^{w}$ itself being possibly non-integrable.

We must ensure the uniformity of convergence with respect to functions $h$ for the integral that is disposed of by means of the Riemann-Lebesgue lemma. This is readily done by approximating the function of $l$ that results in from integration over $|\theta|>\varepsilon$ by a smooth function for each positive $\varepsilon$, the other integral approaching zero uniformly as $\varepsilon \rightarrow 0$.

Acknowledgments. The author wishes to thank the anonymous referees for their valuable suggestions to the original manuscript, which lead the author to revise the paper in several significant ways.

\section{References}

[1] M. Bousquet-Mélou and G. Schaeffer, Walks on the slit plane. Probab. Theor. Relat. Fields 124 (2002) 305-344. MR1939650

[2] Y. Fukai, Hitting distribution to a quadrant of two-dimensional random walk, Kodai Math. Jour. 23 (2000) 35-80. MR1749384

[3] Y. Fukai and K. Uchiyama, Potential kernel for two-dimensional random walk, Ann. Probab. 24 (1992) 1979-1992. MR1415236

[4] H. Kesten, Hitting probabilities of random walks on $\mathbf{Z}^{d}$, Stoch. Proc. Appl. 25 (1987) 165-184. MR0915132

[5] G. Kozma and E. Schreiber, An asymptotic expansion for the discrete harmonic potential, Electronic J.P. 9 (2004) 1-17. MR2041826

[6] E.M. Stein, Singular integrals and differentiable properties of functions, Princeton University Press, 1970. MR0290095

[7] F. Spitzer, Principles of Random Walks, Van Nostrand, Princeton, 1964. MR0171290

[8] K. Uchiyama, Green's functions for random walks on $\mathbf{Z}^{d}$, Proc. London Math. Soc. 77 (1998), 215-240. MR1625467

[9] K. Uchiyama, The hitting distributions of a line for two dimensional random walks, Trans. Amer. Math. Soc. 362 (2010) 2559-2588. MR2584611

[10] K. Uchiyama, The hitting distributions of a half real line for two dimensional random walks. to appear in Ark. Mat. 48 (2010). MR2584611

[11] K. Uchiyama, Random walks on the upper half plane. preprint 